\title{
Transplants of Neurotrophin-Producing Autologous Fibroblasts Promote Recovery of Treadmill Stepping in the Acute, Sub-Chronic, and Chronic Spinal Cat
}

\author{
Alexander J. Krupka, Itzhak Fischer, and Michel A. Lemay ${ }^{1}$
}

\begin{abstract}
Adult cats show limited spontaneous locomotor capabilities following spinal transection, but recover treadmill stepping with body-weight-supported training. Delivery of neurotrophic factors such as brain-derived neurotrophic factor (BDNF) and neurotrophic factor 3 (NT-3) can substitute for body-weight-supported training, and promotes a similar recovery in a shorter period of time. Autologous cell grafts would negate the need for the immunosuppressive agents currently used with most grafts, but have not shown functional benefits in incomplete spinal cord injury models and have never been tested in complete transection or chronic injury models. In this study, we explored the effects of autologous fibroblasts, prepared from the individual cats and modified to produce BDNF and NT-3, on the recovery of locomotion in acute, sub-chronic and chronic fulltransection models of spinal injury. Fourteen female cats underwent complete spinal transection at T11/T12. Cats were separated into four groups: sham graft at the time of injury, and BDNF and NT-3 producing autologous fibroblasts grafted at the time of injury, 2 weeks after injury, or 6 weeks after injury. Kinematics were recorded 3 and 5 weeks after cell graft. Additional kinematic recordings were taken for some cats until 12 weeks post-graft. Eleven of 12 cats with neurotrophin-producing grafts recovered plantar weight-bearing stepping at treadmill speeds from 0.3 to $0.8 \mathrm{~m} / \mathrm{sec}$ within 5 weeks of grafting, whereas control cats recovered poor quality stepping at low speeds only $(\leq 0.4 \mathrm{~m} / \mathrm{sec})$. Further, kinematic measures in cats with grafts were closer to pre-transection values than those for controls, and recovery was maintained up to 12 weeks post-grafting. Our results show that not only are autologous neurotrophin-producing grafts effective at promoting recovery of locomotion, but that delayed delivery of neurotrophins does not diminish the therapeutic effect, and may improve outcome.
\end{abstract}

Keywords: animal studies; locomotor function; neuroplasticity; spinal cord injury

\section{Introduction}

Pontaneous ReCOVERy of treadmill locomotor activity is limited in spinal cats, but treadmill training promotes the development of full-weight-bearing plantar stepping. ${ }^{1,2}$ The bodyweight support training (BWST) originally developed in cats is now used clinically to aid recovery in spinal cord injured (SCI) individuals. $^{3-5}$ Although effective in incomplete SCI, BWST is insufficient for patients with complete motor deficits (American Spinal Injury Association [AISA] A or B) where if fails to restore a functional locomotor activation pattern in the leg muscles. ${ }^{6-8}$

Recent experiments in motor complete SCI individuals have shown that epidural stimulation used in conjunction with treadmill training produces stepping in patients with motor complete injuries. ${ }^{8-11}$ The hypothesized mechanism is that the electrical stimulation elevates the excitability level of the locomotor centers, whose activity is in turn modulated by the leg sensory feedback, producing appropriate patterns of activation for the flexors and extensors of the legs, and even a return of some voluntary control. ${ }^{10}$

We have previously shown that delivering neurotrophins to the injured spinal cord promotes recovery of stepping to levels comparable with those that can be reached with treadmill training. ${ }^{12}$ The combination therapy of neurotrophins and treadmill training was also shown to result in a superior recovery than the one resulting from training or neurotrophin delivery alone. Neurotrophins may thus facilitate re-engaging of the locomotors centers and serve as a substitute or facilitator for treadmill training, ${ }^{13}$ although refinement to the delivery methods is needed before clinical trials are attempted.

In this study, we demonstrate the feasibility of using delayed, autologous grafts for the promotion of stepping in complete spinalinjured cats. Previous graft studies have utilized allografts from donor animals ${ }^{14,15}$ or xenografts ${ }^{12}$ genetically modified to express neurotrophins. Survival of these grafts requires immunosuppression, ${ }^{16,17}$ which is associated which increased cancer risks. ${ }^{18,19}$

${ }^{1}$ Department of Bioengineering, Temple University, Philadelphia, Pennsylvania. ${ }^{2}$ Department of Neurobiology \& Anatomy, Drexel University College of Medicine, Philadelphia, Pennsylvania. 
Autologous grafts present no such risk and can be readily prepared per patient. Delayed delivery of the grafts into a chronic injury model served to verify the efficacy of this treatment for patients with chronic injuries.

\section{Methods}

\section{Subjects and outline}

Fourteen adult domestic shorthaired cats (Liberty Research Inc., Waverly, NY) were used for this study. All animal procedures were conducted under a protocol approved by the Drexel University College of Medicine Institutional Animal Care and Use Committee (IACUC) and in accordance with the National Institute of Health Guide for the Care and Use of Laboratory Animals as well as all United States Department of Agriculture (USDA) regulations governing the use of felines in research.

Cats were acclimated to handling for 1 week before any experimental or training procedures began. Once comfortable with handling, cats were trained to walk on a treadmill for 3-6 weeks until they were capable of uninterrupted, well-behaved walking for $20 \mathrm{~min}$ at speeds ranging from 0.3 to $0.8 \mathrm{~m} / \mathrm{sec}$. After treadmill training, cats were assigned to one of four groups: control (CONTROL, $n=2$ ), acute autologous fibroblast graft (ACUTE, $n=2$ ), sub-chronic autologous fibroblast graft (SUB-CHRONIC, $n=6$ ), or chronic autologous fibroblast graft (CHRONIC, $n=4)$. Figure 1 outlines the experimental protocol. Cell biopsy was performed for each cat in the neurotrophin-treated groups.

Both CONTROL cats underwent a T11-T12 spinal transection followed by a 2 week delay and a sham graft surgery. For sham grafts, the lesion site was debrided as for the other groups, but the cavity was left empty. ACUTE group cats received modified fibroblasts into the lesion site at the time of injury. SUB-CHRONIC cats received the graft 2 weeks after the transection, and CHRONIC cats received the graft 6 weeks after transection. Treadmill recording sessions occurred 3,5 , and in some cases 8 and 12 weeks after grafting.

One cat (SUB-CHRONIC-6) received a graft of cells but was euthanized after 1 week to evaluate graft survival. Biotinylated dextran amine (BDA) tracers were delivered to three cats in the CHRONIC group, as described subsequently.

\section{Fibroblast collection and preparation}

Autologous fibroblasts were obtained for all graft cats via skin biopsy. Four $6 \mathrm{~mm}$ skin punches were obtained from the lower abdomen of the anesthetized animal. The skin punch injuries were closed with 5-0 Prolene surgical sutures.

After collection, skin biopsy samples were cultured in an incubator to allow for expansion and collection of fibroblasts. Cell culturing used a medium of Dulbecco's Modified Eagle Medium (DMEM) with $10 \%$ fetal bovine serum (FBS). When 1,500,000 fibroblasts had been collected, cells were split into two groups and infected via lentivirus with brain-derived neurotrophic factor (BDNF) or neurotrophic factor 3 (NT-3), following a procedure previously described by Blesch. ${ }^{20}$ Briefly, cells were expanded until $\sim 3,000,000$ were available, at which point the cells were split into two plates. A lentiviral vector expressing BDNF or NT-3 was added to each set of cells, resulting in one cell line expressing $\mathrm{BDNF}$, and one expressing NT-3. Green fluorescent protein (GFP) was included in the transgene as a reporter. Following infection,

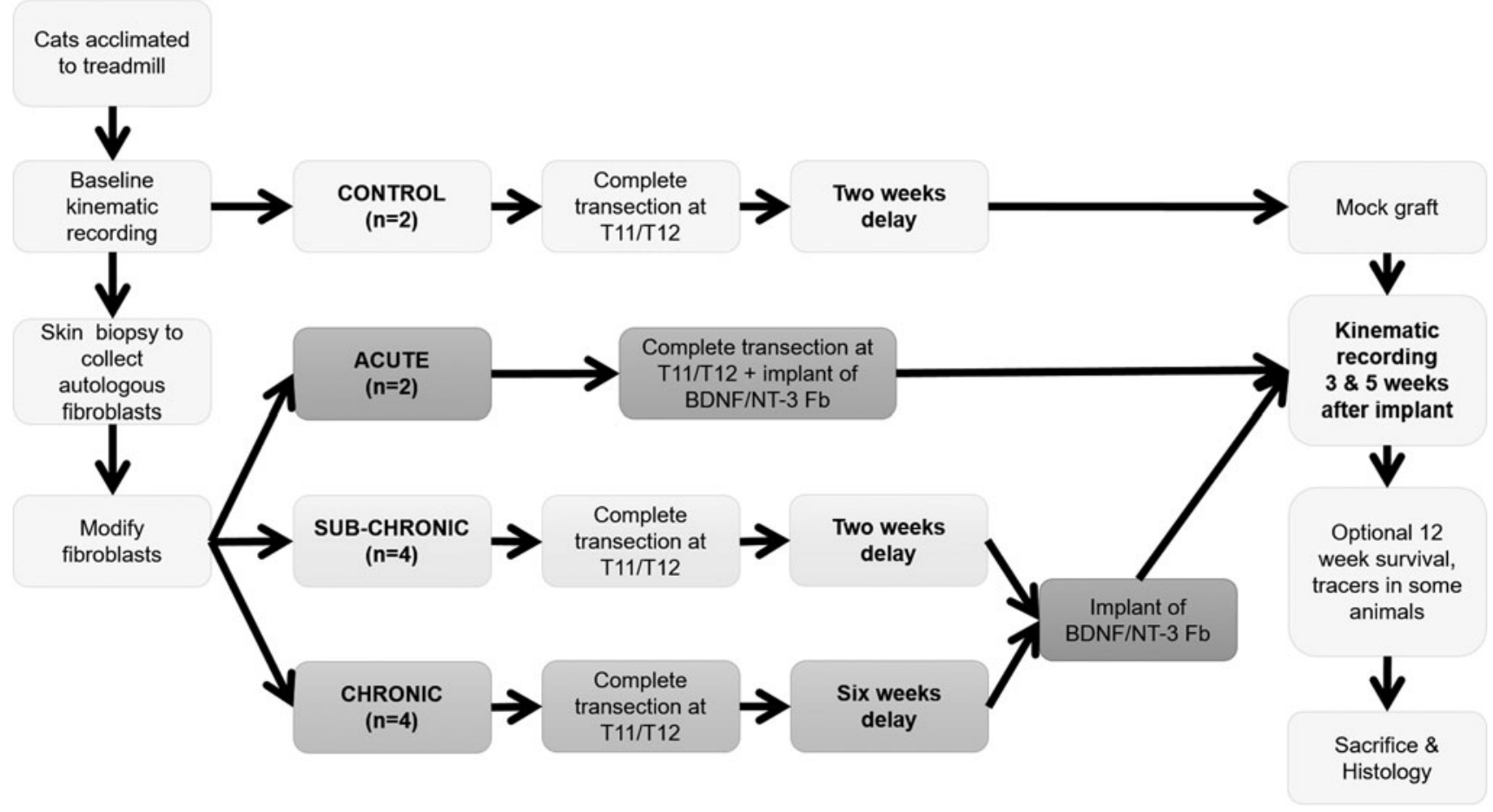

FIG. 1. Experimental timeline. Following treadmill acclimation and acquisition of pre-transection walking data, neurotrophin-treated cats underwent surgery to obtain fibroblast samples. Those fibroblasts were transfected to express brain-derived neurotrophic factor (BDNF) or neurotrophic factor 3 (NT-3) with a green fluorescent protein (GFP) reporter. No fibroblasts were collected in CONTROL animals. All cats received a complete transection at the T11/T12 spinal level. ACUTE group cats received a graft of modified autologous fibroblasts at the time of surgery; other cats received this graft after a delay. CONTROL cats underwent a mock grafting surgery 2 weeks post-transection, when the injury site was debrided as for the neurotrophin- treated groups, but no cells were implanted into the cavity site. CHRONIC group cats had an extra treadmill recording session 5 weeks after injury (1 week before grafting) to assess any natural recovery. Kinematic recordings were made at 3 and 5 weeks post-grafting for all cats. Some animals survived to 12 weeks with extra recording sessions. Histological evaluation was performed after the last kinematic recording for all cats. 
cells were expanded until $\sim 5,000,000$ each of BDNF-infected and NT-3-infected cells were available. To ensure that cells produced effective levels of neurotrophic factors, cells were observed under the microscope for activity of the GFP reporter gene. Before grafting, cell populations were confirmed to have a minimum of $30 \%$ of the cells expressing the reporter. Total preparation time for the graft from collection to the preferred cell count took $\sim 6$ weeks; cells were frozen at $-80^{\circ} \mathrm{C}$ to maintain stocks if necessitated by scheduling. For grafting, cells were suspended in sterile DMEM with 30\% FBS and placed on ice; the supernatant fluid was separated and removed immediately prior to grafting. Any cells remaining after grafting were maintained to acquire supernatant for enzyme-linked immunosorbent assay (ELISA). ELISA was performed with a Human BDNF or Human NT-3 ELISA kit (DuoSet ELISA Development System, Human BDNF Cat No. DY 267 or Human NT-3 Cat No. 248). In some cases (SUB-CHRONIC-6, CHRONIC-2, CHRONIC-4), no cells remained after grafting, and, therefore, no neurotrophin production data were available for those cats.

\section{Spinal transection and graft delivery}

Cats were anesthetized and prepared for surgery in accordance with Drexel University Laboratory Animal Resources policy. A midline incision was made in the skin overlying T9 to L1. Blunt dissection was used to separate the muscle from the spinal vertebrae. Rongeurs were used to remove the dorsal process of the T11 vertebra; the T12 dorsal process was left minimally damaged and served as a landmark for subsequent procedures. Upon exposing the spinal cord, the dura mater overlaying the dorsal cord was carefully opened. Once the dura was opened, xylocaine $(1 \%)$ was applied topically as well as injected $(0.2-0.3 \mathrm{~mL}$ total $)$ into the spinal cord to reduce injury discharge by neurons/axons during transection. The spinal cord was completely severed using microscissors, creating a 3-5 $\mathrm{mm}$ long "pocket" formed by the dura mater and bordered at the rostral and caudal edges by the cut spinal cord.

At the time of grafting, cells were spun down with a tabletop centrifuge for $\sim 20 \mathrm{sec}$. Excess medium was removed with a Hamilton syringe and the cells were again spun down, and excess medium removed. After the second spin, cells were drawn into the Hamilton syringe with $18 \mathrm{G}$ needle and delivered into the dura pocket until it was full, from $6,000,000$ to $10,00,000$ cells depending upon the animal. For SUB-CHRONIC and CHRONIC group cats, as well as untreated injured animals, the cavity was left empty. The dura was sutured closed with 7-0 Prolene in all cases. Following dura closure, muscle and tissue layers were sutured with resorbable 4-0 Vicryl sutures, and the skin closed with 5-0 Prolene.

For delayed graft surgery (SUB-CHRONIC, CHRONIC, and CONTROL groups), the same initial procedure was used. Once the dura was opened, the lesion area was debrided, removing scar tissue and taking care not to disturb the uninjured rostral and caudal ends. The lesion was cleared until a cavity $3-5 \mathrm{~mm}$ in length was available, with no scar or nervous tissue visible within the dura. In SUBCHRONIC and CHRONIC cats, cells were grafted into this cavity as described. CONTROL cats received no graft or injection at the site; the cavity remained empty. After grafting the dura, muscle, and skin layers were closed as described.

\section{Treadmill acclimation and locomotor recording}

Prior to spinalization, cats were acclimated to the treadmill. Once acclimated, treadmill training sessions lasted $20 \mathrm{~min}$, and were performed once a day, five times a week. The cat was placed inside a clear plexiglass box and given food rewards to maintain forward attention while walking. The treadmill was capable of operation at speeds from 0.2 to $0.8 \mathrm{~m} / \mathrm{sec}$. All cats were trained to locomote at all speeds from 0.3 to $0.8 \mathrm{~m} / \mathrm{sec}$ in $0.1 \mathrm{~m} / \mathrm{sec}$ increments. Before transection, cats performed quadrupedal stepping; after transection the forepaws rested on a stationary platform suspended $1 \mathrm{~cm}$ above the surface during treadmill sessions. Cats were not trained following spinalization, and only stepped on the treadmill during recording sessions. Previous work has indicated that treadmill stepping every 2 weeks is not sufficient to induce a training effect on locomotion. ${ }^{12}$

Data collection was handled by the Vicon Nexus (Vicon Industries, Inc. Hauppauge NY) recording software. Collected data included positions for each of 11 skeletal markers and a video recording (30 fps) of each session. Figure 2 shows the locations of the

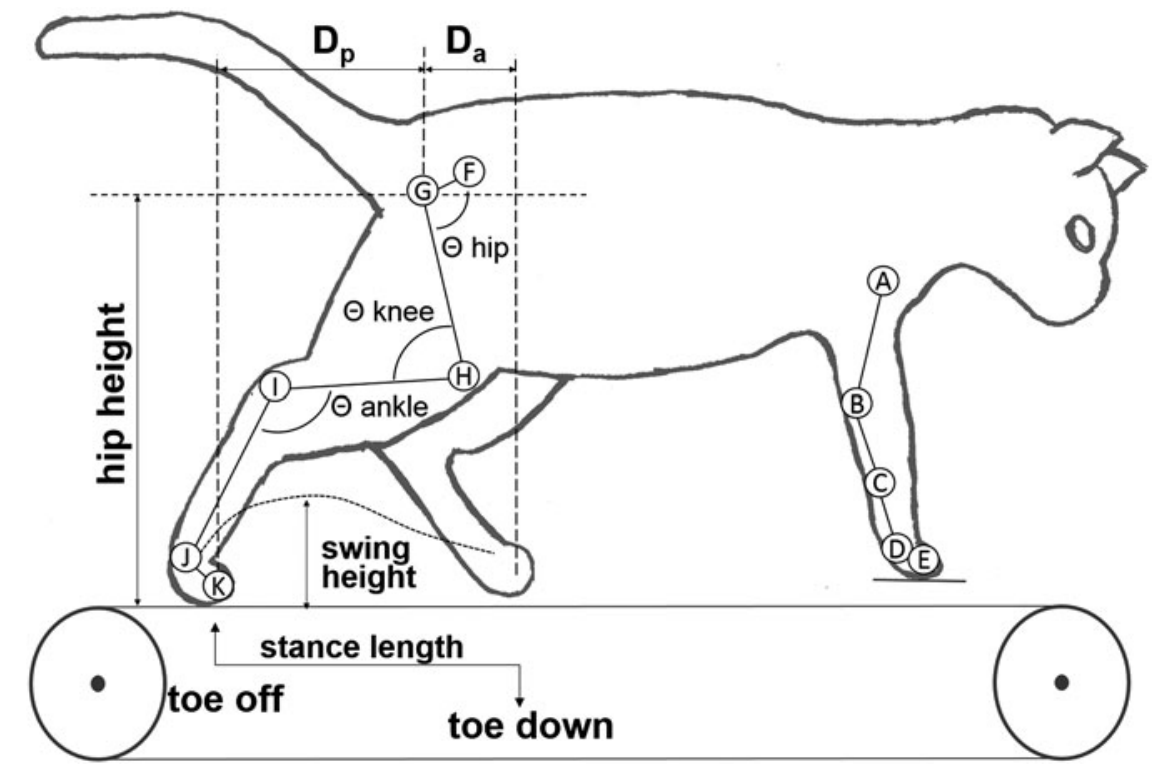

FIG. 2. Diagram of the joint marker locations and kinematic parameters extracted. Reflective markers were temporarily attached to the skin over the (A) humeral head, (B) elbow, (C) lateral malleolus, (D) metacarpophalangeal joint, (E) second forelimb digit, (F) pelvis, (G) femoral head, (H) knee, (I) ankle, (J) metatarsophalangeal joint, and (K) second digit of the hindlimb. Stance length, swing height, $\mathrm{D}_{\mathrm{a}}$ (horizontal displacement of the foot relative to hip at toe-down), $\mathrm{D}_{\mathrm{p}}$ (horizontal displacement of the foot relative to hip at liftoff), hip height, and hip, knee and ankle angles are illustrated. 
kinematic markers. Each cat was evaluated on the ability to locomote at all speeds from 0.3 to $0.8 \mathrm{~m} / \mathrm{sec}$; to be considered capable of stepping, a cat had to perform 10 consecutive plantar weight-bearing steps at each speed and the speeds below it. ${ }^{.2,21}$ Marker data were exported and broken into individual steps using custom MATLAB scripts (The MathWorks Inc., Natick MA) and then individually analyzed. Kinematic parameters were calculated for each step with Igor Pro (Wavemetrics, Inc. Lake Oswego OR), and exported to SPSS (IBM Corp., Armonk NY) for statistical analyses.

Baseline treadmill kinematics were recorded for all cats before transection. Additionally, stepping ability in CHRONIC cats was evaluated 5 weeks after injury; that is, 1 week before grafting. Posttransection recording sessions occurred 3 and 5 weeks after grafting, and some animals in the CHRONIC graft group underwent additional recording 8 and 12 weeks after grafting. The experimenter held the cat at the base of the tail to provide balance support during recording sessions and perineal stimulation was delivered to maximize locomotor output in each of the animals, as in previous studies. ${ }^{12,21}$ Only perineal stimulation sufficient to initiate stepping was applied, as excessive stimulation can interrupt stepping or cause aberrant patterns. Actual treadmill recording time was $\sim 1 \mathrm{~min}$ at each speed and each $1 \mathrm{~min}$ of recording constituted a trial that produced a minimum of 10 steps for analysis in all cases.

For one animal (CHRONIC-1), an instrumented treadmill was available to record ground reaction forces (GRFs). The instrumented treadmill consisted of two independent treadmills mounted side by side on independent force plates (Kistler Type 9257BA; Kistler Instrument Corp., Winterthur, Switzerland). The divider used to prevent scissoring of the legs in spinalized animals separated the two belts, and each treadmill sub-assembly could record fore-aft (anteroposterior), mediolateral, and vertical forces individually for each hindlimb. GRFs were normalized to the mass of the cat at the time of the recording for comparison with previous reports on GRFs during walking or stance in able-bodied and spinalized felines.

\section{Kinematic and GRF analysis}

Horizontal and vertical positions for each of the 11 markers were exported from Vicon into a text file and imported into MATLAB. Depth data were discarded for all analyses. Custom MATLAB scripts separated the trials into component steps and exported marker positions for each step as text files for further analysis. Steps were imported into Igor Pro to obtain quantifications of stepping parameters. The mean lateral movement of the hip marker was calculated for all steps in the trial; steps with movement $>3$ standard deviations from the mean were automatically excluded from analysis. To determine the percentage of steps that were plantar, a $20 \mathrm{sec}$ video clip from each animal was observed at half speed. The number of plantar- and dorsi-steps were recorded and summed within each group to calculate the percentage of steps taken in which the paw was plantar.

The positions of the elbow and knee markers were triangulated based on anatomical measurements to correct for skin slippage. ${ }^{22}$ Marker positions were used to obtain a number of kinematic parameters: stance length $(\mathrm{mm})$, swing height $(\mathrm{mm}), \mathrm{D}_{\mathrm{a}}(\mathrm{mm}$, horizontal displacement of the foot relative to hip at toe-down), $\mathrm{D}_{\mathrm{p}}$ ( $\mathrm{mm}$, horizontal displacement of the foot relative to hip at liftoff). Using the position of the toe marker, the duration of each step was calculated, as well as the length of each stance phase. Additional analysis also produced minimum, maximum, and range values for the hip, knee, and ankle joints. Visual depictions and descriptions of these measures are included in Figure 2. These output measures were the primary dependent variables for subsequent analysis.

The kinematic measures for each plantar step were averaged over a trial to produce a mean value. Analysis was then performed at the trial level, using these mean values from each trial. For posttransection kinematics recording sessions, we analyzed one trial per speed, per time point for each cat. Pre-transection data sometimes included multiple trials (two to four) at the same speed (all pretransection recordings were grouped as one time point).

SPSS was used for all statistical analyses. Values for stance length, swing height, hip height, $\mathrm{D}_{\mathrm{a}}$, and $\mathrm{D}_{\mathrm{p}}$ were normalized to pretransection values; subsequent analyses involving these variables were performed on these indices. The average values of each variable per trial were used for analysis.

Mixed model analysis was used with kinematic measures (stance length index, swing height index, hip height index) as the dependent variables, and group, speed, and weeks post-grafting as fixed factors. Cat was included as a subject level factor whereas trial, speed, and time post-grafting (including the pre-transection/baseline time point) were modeled as repeated factors. When multiple factors were included in an analysis (e.g., speed and group), a full-factorial model including main effects (e.g., speed and groups) and all possible interaction terms (e.g., speed by group) was used.

Post-hoc comparison of main effects was performed using the Bonferroni post-hoc analysis. All tests were performed at the 0.05 significance level. Unless otherwise stated, all reported values are the estimated marginal means \pm standard error.

All GRF data was analyzed using routines written in MATLAB or Python (Python Software Foundation, www.python.org) language. Treadmill resonance frequencies were filtered out using discrete wavelet transform (DWT) SureShrink denoising method. Baseline correction was applied to the data by calculating the mean of force data points during the swing phase of the gait cycle, and subtracting it from the entire data set. The stance onset, offset, and peak vertical force points of each step in the data were then identified from the vertical ground reaction forces by manual user selection. Stance time was normalized from 0 (onset) to $100 \%$ (offset), and ground reaction force in all three axes during stance was normalized to the mass of the cat at the time of the recording session. The normalized forces were then averaged across all steps to produce a mean force profile across stance time for all three Cartesian directions (see Fig. 3).

\section{Tracer delivery}

Three cats in the CHRONIC group underwent tracer delivery. Vertebral bone rostral and caudal to the lesion was removed to provide sites for tracer injection. In one cat, tracer injection occurred only rostral to the lesion, whereas in two cats, different tracers were injected rostral and caudal to the graft site. The tracers used were fluorescent dextran-conjugated micro-ruby (3000MW; Life Technologies, Carlsbad CA) and micro-emerald (3000MW; Life Technologies). Tracer was diluted in sterile saline at a concentration of $100 \mathrm{mg} / \mathrm{mL}$. Hamilton syringes mounted to a stereotaxic frame were equipped with a $27 \mathrm{G}$ needle and used to deliver the tracers. The dura was pierced at the injection site with a $25 \mathrm{G}$ needle or microscissors, and the needle of the Hamilton syringe was guided down into the spinal cord. Approximately $15 \mu \mathrm{L}$ of tracer was injected per injection site, distributed at several depths from $1000 \mu \mathrm{m}$ to $3000 \mu \mathrm{m}$, extending from the left to the right dorsal root entry zones. Injection at each location was performed over $20 \mathrm{~min}$ to ensure that the tracer was injected slowly and completely. After tracer delivery, the dura was closed with 7-0 Prolene, the muscle and fascia layers were closed with resorbable 4-0 Vicryl sutures, and the skin was closed with 5-0 Prolene.

\section{Tissue processing and histology}

After the final kinematic recording session, cats were anesthetized and transcardially perfused with $500 \mathrm{~mL}$ chilled physiological saline followed by $1 \mathrm{~L}$ of $4 \%$ paraformaldehyde in phosphate buffer (PB). The spinal cord was removed and postfixed in $4 \%$ paraformaldehyde. Tissue was cryoprotected in $30 \%$ sucrose after post-fix. 
A

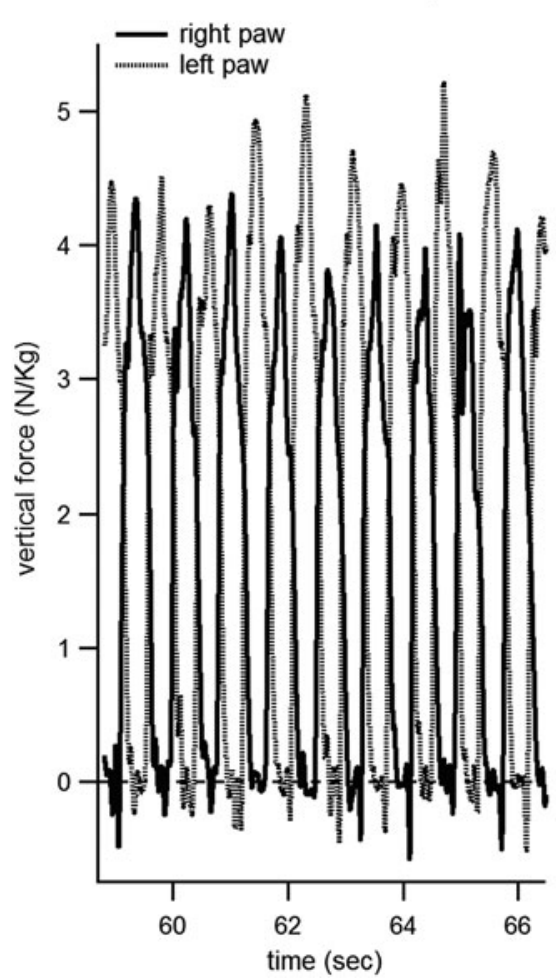

B

average forces during stance

for all three cartesian directions
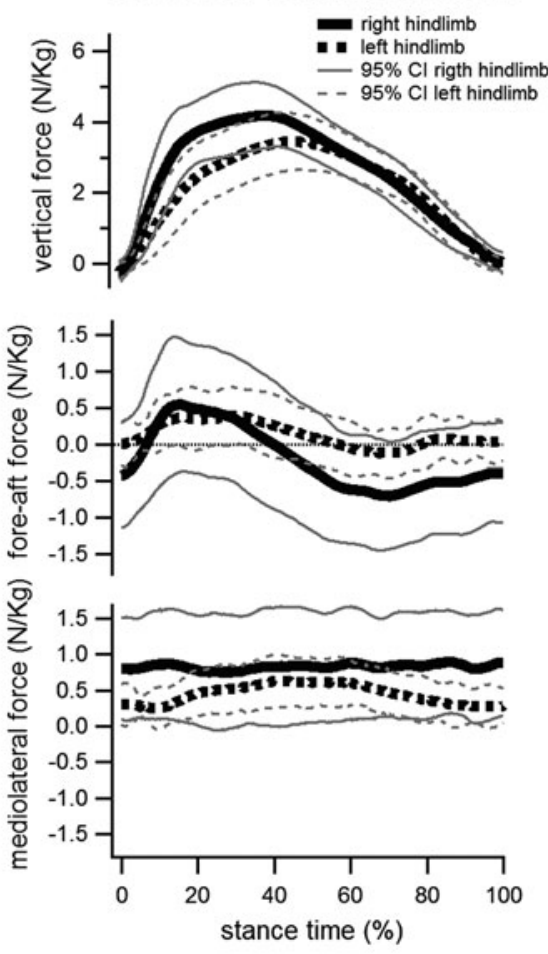

FIG. 3. Normalized ground reaction forces in one cat (CHRONIC-1) at a walking speed of $0.4 \mathrm{~m} / \mathrm{sec} 12$ weeks after grafting. The left panel shows the right and left vertical ground reaction forces over $\sim 10$ steps. Normalized peak vertical force reached $>4 \mathrm{~N} / \mathrm{kg}$ for both hindlimbs during each step over that period. Panels on the right show the means and $95 \%$ confidence intervals of the vertical, fore-aft (or anteroposterior), and mediolateral forces over the stance period (0-100\%). The average peak normalized vertical forces over 163 steps were $3.4 \mathrm{~N} / \mathrm{kg}$ for the left hindlimb and $4.2 \mathrm{~N} / \mathrm{kg}$ for the right hindlimb. Fore-aft forces were initially positive (indicating braking), until $\sim 40 \%$ of the stance phase, when forces turned negative (indicating propulsion). Mediolateral forces did not show the lateral push seen in able-bodied cats, ${ }^{2,25,64,65}$ indicative of the lack of balance control in spinal animals.

After cryoprotection, tissue was sectioned into $40 \mu \mathrm{m}$ slices in the horizontal or lateral planes for evaluation.

Fluorescent images of the graft site and lumbar spinal cord were taken to locate the grafted fibroblasts via the GFP reporter. To assist in locating the cells, some slices were used for immunohistochemical staining for GFP. Axonal sprouting and state of the lesion site were visualized by Nissl-myelin staining. Nissl-myelin stained sections were used to assess completeness of the injury. Sections with fluorescent tracer were not stained with Nissl-myelin but were dehydrated and cover-slipped with Fluorsave (EMD Millipore, Temecula CA) mounting medium. Nissl-myelin sections were imaged with a color camera, whereas fluorescent sections were obtained using a gray scale camera. Microsoft Image Composite (Microsoft, Redmond WA) editor was used to stich adjacent images and provide a more complete view of the tissue.

To determine the location and spread of the graft after delivery, anti-GFP antibodies were used to locate grafted fibroblasts. Tissue used for immunohistological evaluation was cut into $20 \mu \mathrm{m}$ thick sections in the horizontal or sagittal planes. Slices were mounted on slides and ringed with rubber cement to form pools to contain antibody solutions and reagents. Tissue was washed three times in $0.1 \mathrm{M} \mathrm{PB}$ ( $\mathrm{pH} 7.4$ ), after which the tissue was permeabilized in $0.1 \mathrm{M}$ PB with a $0.3 \%$ solution of Triton- $X$ then rinsed. Tissue was blocked for $1 \mathrm{~h}$ in a $10 \%$ goat serum PB solution at room temperature. Tissue was then incubated overnight with the primary antibody diluted in a solution of $2 \%$ goat serum/phosphate-buffered saline (PBS) with $0.2 \%$ Triton. The primary antibody used was against GFP (1:1500, Invitrogen, Carlsbad CA), to locate the grafted fibroblasts. The following day, sections were washed in PBS and incubated with the fluorescent secondary antibody (1:400, Jackson Immuno Research Labs, West Grove PA) in 2\% goat serum for $2 \mathrm{~h}$ at room temperature in the dark. Finally, the slides were rinsed in PBS and cover-slipped using an aqueous mounting medium, Vectashield (Vector Laboratories, Burlingame CA).

\section{Results}

A total of 14 adult female domestic shorthaired cats were utilized in this study. Of these 14 cats, kinematics were collected for 13, because one cat (SUB-CHRONIC-4) was euthanized early for histological analysis. All cats were capable of stepping at each treadmill speed from 0.3 to $0.8 \mathrm{~m} / \mathrm{sec}$ prior to injury.

\section{Post-injury stepping}

CONTROL cats were only capable of limited stepping after injury. Three weeks post-transection, one CONTROL cat was capable of stepping at a maximum speed of $0.4 \mathrm{~m} / \mathrm{sec}$, whereas the other was incapable of stepping at any of the speeds tested. By 5 weeks, both CONTROL cats could perform 10 consecutive plantar weight-bearing steps $\leq 0.4 \mathrm{~m} / \mathrm{sec}$, although the majority of steps were on the dorsal surface of the paw. None of the CONTROL cats were capable of 10 consecutive plantar weight-bearing steps at speeds $>0.4 \mathrm{~m} / \mathrm{sec}$ post-transection. Qualitatively, stepping in the CONTROL group was of a lower overall value than that seen in 
Table 1. Maximum Treadmill Stepping Speed at Key Time Points

\begin{tabular}{llcc}
\hline & \multicolumn{3}{c}{ Maximum treadmill speed } \\
\cline { 2 - 4 } Cat & $\begin{array}{c}\text { Post-injury } \\
\text { pre-graft }\end{array}$ & $\begin{array}{c}\text { 3 Weeks } \\
\text { post-graft }\end{array}$ & $\begin{array}{c}5 \text { Weeks } \\
\text { post-graft }\end{array}$ \\
\hline ACUTE-1 & N/A & $0.3 \mathrm{~m} / \mathrm{sec}$ & $0.8 \mathrm{~m} / \mathrm{sec}$ \\
ACUTE-2 & N/A & $0.8 \mathrm{~m} / \mathrm{sec}$ & $0.8 \mathrm{~m} / \mathrm{sec}$ \\
SUB-CHRONIC-1 & N/A & $0.6 \mathrm{~m} / \mathrm{sec}$ & $0.8 \mathrm{~m} / \mathrm{sec}$ \\
SUB-CHRONIC-2 & N/A & ---- & ---- \\
SUB-CHRONIC-3 & N/A & $0.8 \mathrm{~m} / \mathrm{sec}$ & $0.8 \mathrm{~m} / \mathrm{sec}$ \\
SUB-CHRONIC-4 & N/A & $0.8 \mathrm{~m} / \mathrm{sec}$ & $0.8 \mathrm{~m} / \mathrm{sec}$ \\
SUB-CHRONIC-5 & N/A & $0.8 \mathrm{~m} / \mathrm{sec}$ & $0.8 \mathrm{~m} / \mathrm{sec}$ \\
SUB-CHRONIC-6 & & No kinematics & \\
CHRONIC-1 & ----- & $0.6 \mathrm{~m} / \mathrm{sec}$ & $0.8 \mathrm{~m} / \mathrm{sec}$ \\
CHRONIC-2 & 0.4 m/sec & $0.8 \mathrm{~m} / \mathrm{sec}$ & $0.8 \mathrm{~m} / \mathrm{sec}$ \\
CHRONIC-3 & 0.3 m/sec & $0.7 \mathrm{~m} / \mathrm{sec}$ & $0.8 \mathrm{~m} / \mathrm{sec}$ \\
CHRONIC-4 & ND & ND & $0.8 \mathrm{~m} / \mathrm{sec}$ \\
CONTROL-1 & ND & ---- & $0.4 \mathrm{~m} / \mathrm{sec}$ \\
CONTROL-2 & ND & $0.4 \mathrm{~m} / \mathrm{sec}$ & $0.4 \mathrm{~m} / \mathrm{sec}$ \\
\hline
\end{tabular}

Maximum treadmill walking speeds for cats in the study at the time points tested. Dashes (-) indicate an inability to perform 10 consecutive plantar weight-bearing steps at any speed, "ND" indicates that data was not collected for a time point, and "N/A" indicates that the time point is not applicable to a particular group. Only one neurotrophin treated cat (SUBCHRONIC 2) was incapable of walking at any treadmill speed at any time point. Stepping ability of CHRONIC cats was recorded 1 week prior to grafting, to quantify any spontaneous recovery at 5 weeks post-transection. Minimal stepping ability was present at this time point, and only two cats were capable of any stepping at low treadmill speeds $(\leq 0.4 \mathrm{~m} / \mathrm{sec})$ and with poor weight support. At 3 weeks post grafting, half of the CHRONIC cats recovered stepping at the maximum treadmill speed $(0.8 \mathrm{~m} / \mathrm{sec})$, whereas the remaining half recovered some stepping at lower speeds. All graft cats except SUB-CHRONIC 2 were capable of full plantar weight- bearing stepping by 5 weeks after grafting at all tested treadmill speeds $(0.3-0.8 \mathrm{~m} /$ $\mathrm{sec}$ ). Recovery in the control cats was limited (low walking speeds and poor quality of stepping, with most steps taken on the dorsal aspect of the foot). graft cats, and it was difficult to isolate 10 consecutive plantar weight-bearing steps from the video recordings in the control group. At least $95 \%$ of the steps observed in the ACUTE, SUBCHRONIC, and CHRONIC groups showed plantar placement of the paw, whereas only $37 \%$ of observed steps were plantar in the CONTROL group.

ACUTE cats readily recovered plantar weight-bearing treadmill stepping. By 3 weeks after injury, one ACUTE cat (ACUTE-2) was capable of plantar, weight-bearing stepping at all tested speeds from 0.3 to $0.8 \mathrm{~m} / \mathrm{sec}$. By 5 weeks after injury, both ACUTE cats were capable of plantar weight-bearing stepping at all speeds from 0.3 to $0.8 \mathrm{~m} / \mathrm{sec}$. The quality of stepping in these cats was superior to that of the CONTROL cats, with ACUTE cats readily performing $\geq 10$ consecutive plantar weight-bearing steps. Three of five SUBCHRONIC cats recovered stepping up to $0.8 \mathrm{~m} / \mathrm{sec} 3$ weeks after grafting, and by 5 weeks after grafting, four SUB-CHRONIC cats were performing 10 plantar weight-bearing steps at all speeds from 0.3 to $0.8 \mathrm{~m} / \mathrm{sec}$. However, one cat (SUB-CHRONIC-2) did not recover any locomotor capability at any time point.

Two cats in the CHRONIC group were incapable of stepping at any speed 5 weeks post-transection/1 week pre-grafting, whereas two could step only at low speeds $(\leq 0.4 \mathrm{~m} / \mathrm{sec})$. At the 3 week postgrafting evaluation, one cat was capable of stepping at all speeds from 0.3 to $0.8 \mathrm{~m} / \mathrm{sec}$, two other cats could only step at lower speeds $(\leq 0.4 \mathrm{~m} / \mathrm{sec})$, and the final cat was incapable of stepping at any speed. By 5 weeks post-grafting, however, all CHRONIC cats were capable of plantar, weight-bearing stepping at speeds ranging from 0.3 to $0.8 \mathrm{~m} / \mathrm{sec}$.

Table 1 outlines the maximum treadmill speed at which the animal could perform 10 consecutive plantar weight-bearing steps at the 3 and 5 week time points for each cat. Detailed kinematics of locomotion are discussed subsequently.

Detailed kinematics of locomotion for all cats were analyzed with a mixed model (see Methods section) for the 5 weeks postgrafting time point. CONTROL cats were capable of 10 consecutive plantar steps at $0.4 \mathrm{~m} / \mathrm{sec}$, although most steps were taken on the dorsal surface of the paw. All graft cats were capable of plantar weight-bearing stepping at speeds from 0.3 to $0.8 \mathrm{~m} / \mathrm{sec}$. Figure 4

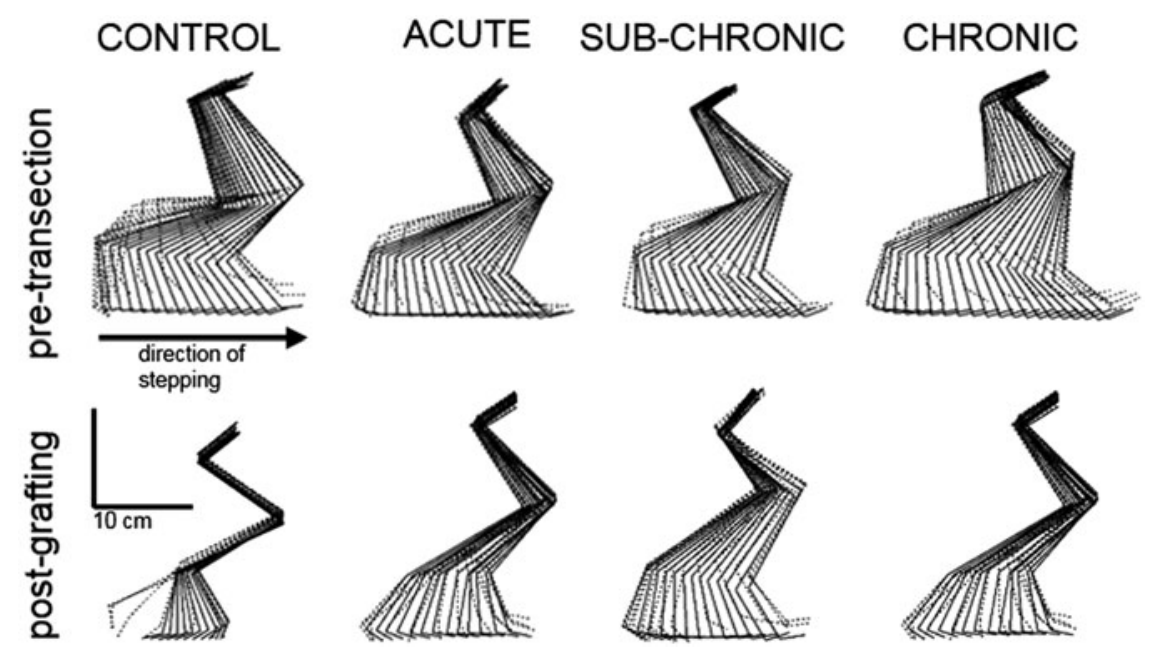

FIG. 4. Stick figure representation of hindlimb kinematics before transection and 5 weeks post-grafting. An exemplar step for one cat from each experimental group is presented. Each stick figure is a selection of frames (displayed at $60 \mathrm{~Hz}$; original recording at $300 \mathrm{~Hz}$ ) during a single step on the treadmill at $0.4 \mathrm{~m} / \mathrm{sec}$ (stance is represented as solid lines, while swing is represented as dotted lines). The step for the CONTROL cat post-transection is representative of the majority of steps recorded in the CONTROL cats, and shows dorsistepping during the stance phase and dragging during the swing phase. 
displays pre- and post-transection steps from the CONTROL, ACUTE, SUB-CHRONIC, and CHRONIC groups 5 weeks after graft at a speed of $0.4 \mathrm{~m} / \mathrm{sec}$. Neurotrophin-treated animals showed consistent plantar placement of the paw on the treadmill with good clearance above the treadmill surface during swing, whereas control cats showed more dragging and dorsal placement of the paw.

Figure 5 shows several kinematic parameters for each group 5 weeks after grafting when walking at $0.4 \mathrm{~m} / \mathrm{sec}$. A linear mixed model indicated a significant effect of experimental group on the recovery of stance length post-transection $(p=0.012)$. The normalized stance length of the plantar steps taken by CONTROL cats $(64 \pm 6 \%)$ was significantly lower than that seen in SUB-CHRONIC $(96 \pm 4 \%)$ and CHRONIC (94 $\pm 5 \%)$ graft cats. Mean stance length index in ACUTE cats was lower than that seen in the SUBCHRONIC and CHRONIC graft cats and higher than that seen in CONTROL cats; however, the ACUTE group was not significantly different from any other group. The SUB-CHRONIC and CHRONIC group cats recovered stance length to pre-transection levels.
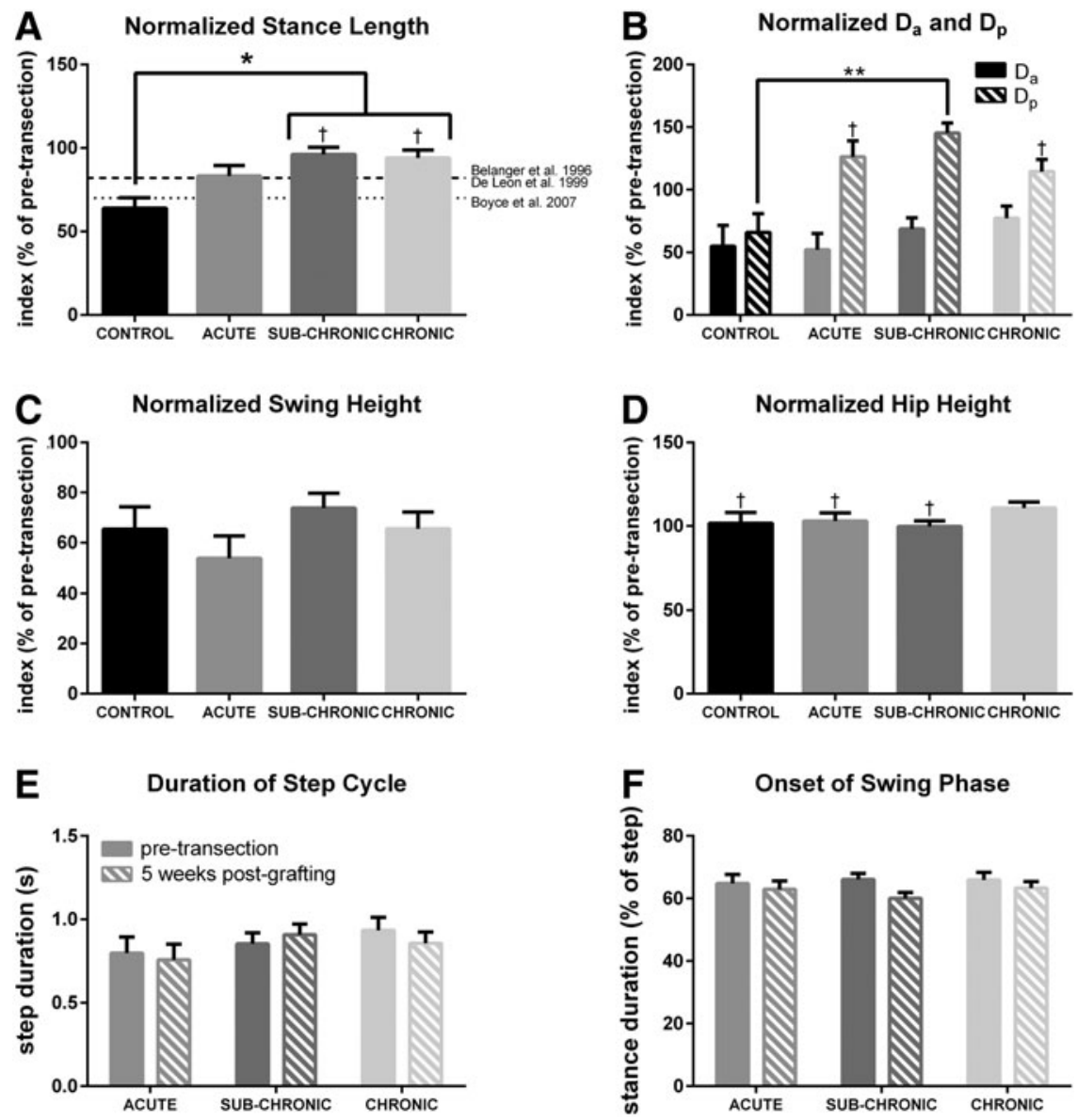

FIG. 5. Analysis of various kinematic parameters at a speed of $0.4 \mathrm{~m} / \mathrm{sec} 5$ weeks post-fibroblast grafting with respect to pretransection. Stance length, $\mathrm{D}_{\mathrm{a}}$ (horizontal displacement of the foot relative to hip at toe-down), $\mathrm{D}_{\mathrm{p}}$ (horizontal displacement of the foot relative to hip at liftoff), swing height, and hip height are normalized to pre-transection means (100\%). (A) There was a significant effect of group on recovery of stance length (linear mixed model, $p=0.012$ ), with the SUB-CHRONIC and CHRONIC groups recovering to significantly higher levels than the CONTROL group. Recovery in the ACUTE group was not significantly different from that in any other group, and was comparable with prior results with body-weight-supported locomotor training or xenografts indicated by the labeled lines. Recovery in stance length index obtained with body-weight-supported locomotor training is indicated by a dashed-dotted line, ${ }^{1,32}$ whereas the recovery obtained with xenografts producing neurotrophins with no locomotor training is indicated by a dotted line. ${ }^{12}$ (B) $\mathrm{D}_{\mathrm{a}} 5$ weeks after grafting was lower than pre-transection in all cats $(95 \%$ confidence interval [CI] did not contain pretransection mean); however, there was no significant difference among groups (linear mixed model, $p=0.444$ ). There was a high variability in $\mathrm{D}_{\mathrm{p}}$ among groups after grafting, as well as a significant effect of group (linear mixed model, $p=0.006$ ). $\mathrm{D}_{\mathrm{p}}$ in the SUBCHRONIC group was significantly higher than in the CONTROL group. The graft groups trended to have a higher $\mathrm{D}_{p}$ than pretransection, whereas the CONTROL group trended below pre-transection. For the ACUTE and CHRONIC groups, the pre-transection mean was within the $95 \%$ CI. (C) There was no significant effect of group on swing height (linear mixed model, $p=0.369$ ). No group was significantly different from pre-transection. (D) There was no significant effect of group on hip height (linear mixed model, $p=0.247$ ). The CHRONIC group, however, had a significantly higher hip height compared with pre-transection. (E) Step duration was unchanged for the ACUTE, SUB-CHRONIC, and CHRONIC groups after transection and grafting. (F) Similarly, there was no change in stance duration after transection and grafting. The limited number of high-quality post-transection steps precluded analysis of step and stance duration in control cats. Error bars indicate the standard error of the mean (SEM). * $p<0.05$; ** $p<0.01$; †pre-transection group mean $(100 \%)$ falls within the group's $95 \%$ CI post-injury. 
Swing height was also analyzed for all cats 5 weeks post-grafting at $0.4 \mathrm{~m} / \mathrm{sec}$, and we found no significant effect of group ( $p=0.369$ ). Overall, there was high variability in swing height indices among the graft groups, ranging from $54 \pm 9 \%$ in the ACUTE group, to $66 \pm 7 \%$ in the CHRONIC group and $74 \pm 6 \%$ in the SUB-CHRONIC group.

To obtain a clearer picture of the changes post-transection in the horizontal paw position, $\mathrm{D}_{\mathrm{a}}$ (maximal toe distance anterior to the hip at toe-down) and $\mathrm{D}_{\mathrm{p}}$ (maximal toe distance posterior to the hip at toe-off) indices were analyzed with a mixed model. Figure $5 \mathrm{~b}$ reports $D_{a}$ and $D_{p}$ indices for the ACUTE, SUB-CHRONIC, and CHRONIC groups. There was no significant main effect of group on $\mathrm{D}_{\mathrm{a}}(p=0.444)$, and $\mathrm{D}_{\mathrm{a}}$ was significantly lower post-transection in all groups $(\mathrm{CONTROL}=54.9 \pm 17 \%, \mathrm{ACUTE}=52+13 \%$, SUB$\mathrm{CHRONIC}=69 \pm 9 \%, \mathrm{CHRONIC}=77 \pm 10 \% ; 95 \%$ confidence interval does not include pre-transection mean in any group).
The post-transection and grafting recovery of $D_{p}$ was more variable. The CONTROL group $\mathrm{D}_{\mathrm{p}}$ index was significantly lower than pre-transection $(66 \pm 15 \%)$. The ACUTE and CHRONIC group $\mathrm{D}_{\mathrm{p}}$ indices did not significantly deviate from pre-transection at $126 \pm 13 \%$ and $115 \pm 10 \%$, respectively. The SUB-CHRONIC group $\mathrm{D}_{\mathrm{p}}$ index increased significantly to $145 \pm 8 \%$. This increase resulted in a significant difference in $\mathrm{D}_{\mathrm{p}}$ indices between the SUBCHRONIC and CONTROL groups $(p=0.004)$. Overall, $\mathrm{D}_{\mathrm{a}}$ decreased after injury, whereas there was a trend of increasing $D_{p}$ in graft animals. Therefore, the hindlimbs tended to extend further back before swing onset, and not extend as far forward at toe down.

Figure 3 shows the GRFs for cat CHRONIC-1 12 weeks after grafting. The animal produced peak normalized vertical forces $>4 \mathrm{~N} / \mathrm{kg}$ over multiple bouts of locomotion (Fig. 3, left panel). Because the hindlimbs support $\sim 40 \%$ of the body mass during walking
A

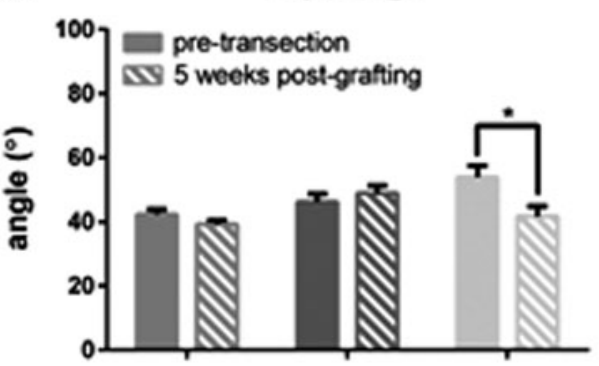

Knee Range

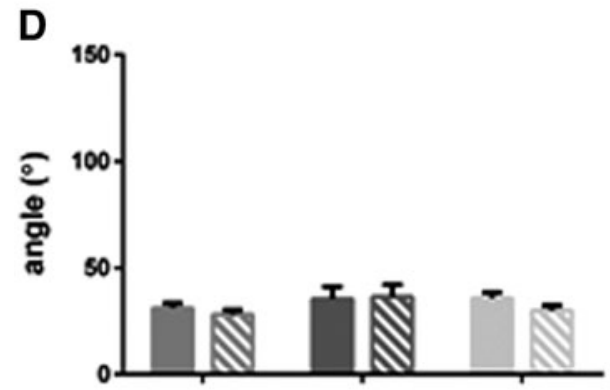

Ankle Range

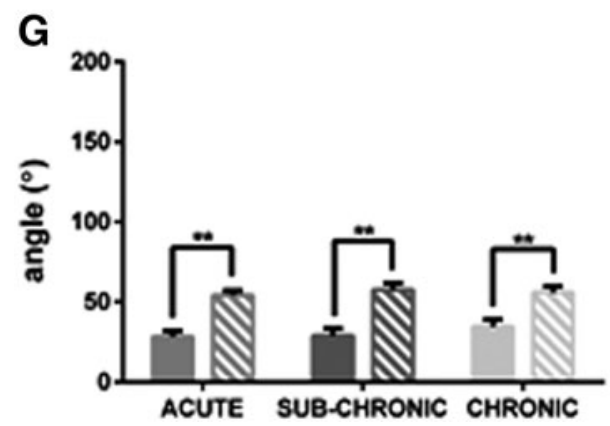

B Hip Maximum Angle

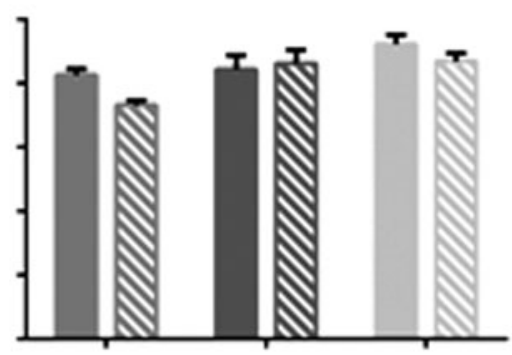

Knee Maximum Angle

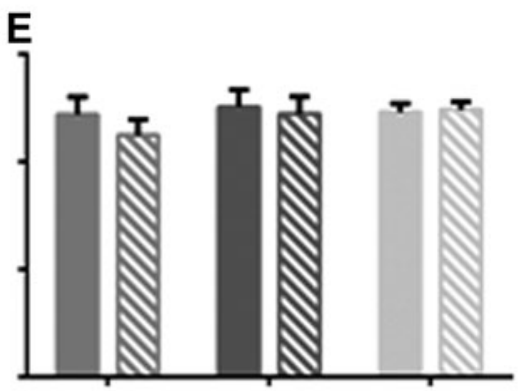

Ankle Maximum Angle

$\mathbf{H}$

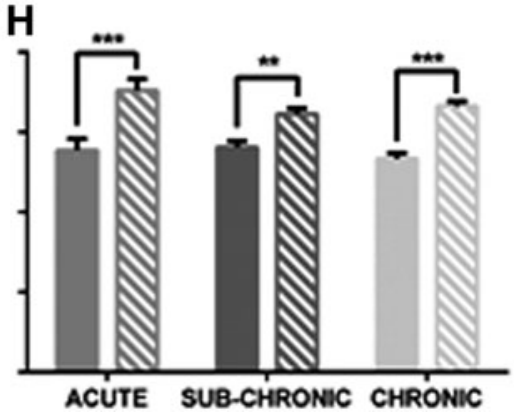

C Hip Minimum Angle

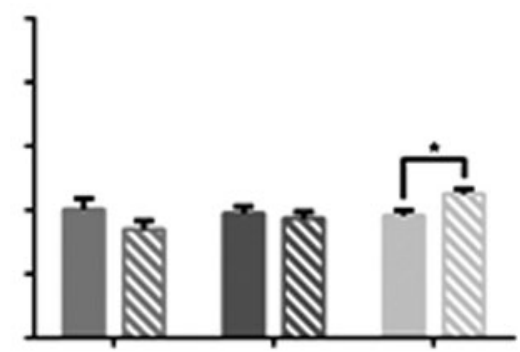

Knee Minimum Angle

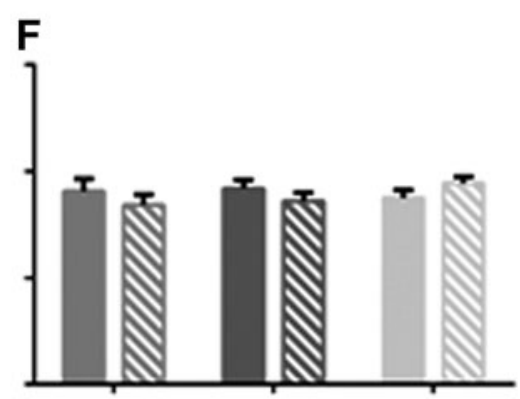

Ankle Minimum Angle

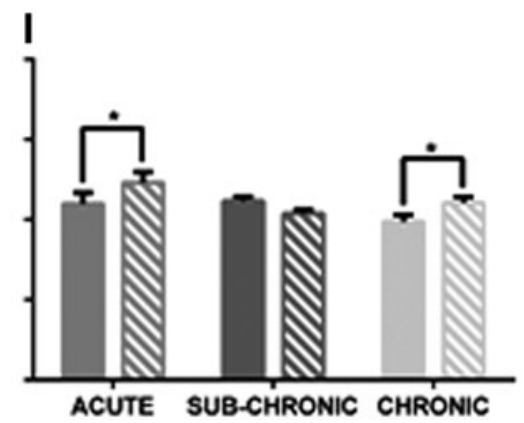

FIG. 6. Comparison of ankle angle measurements before injury and 5 weeks post- grafting during treadmill locomotion at $0.4 \mathrm{~m} / \mathrm{sec}$. (A-C) Hip range significantly decreased after transection in CHRONIC cats, a result of diminished hip flexion. Hip characteristics were unchanged in the other groups. (D-F) Knee angles were consistent pre- and post-transection in all groups assessed. (G-I) Ankle range increased significantly after transection in the neurotrophin graft cats (ACUTE, SUB-CHRONIC, CHRONIC). The maximum angle of the ankle increased significantly at the later time point in all cats. The ankle minimum angle increased significantly in ACUTE and CHRONIC cats, although the magnitude of this increase was much smaller than the change in maximum angle or in overall range. There were not a sufficient number of usable steps in CONTROL cats for inclusion in angle analysis. Error bars indicate SEM. $* p<0.05 ; * * p<0.01 ; * * * p<0.001$. 
or stance in cats, ${ }^{23-25}$ the required vertical force for full weight bearing is roughly $4 \mathrm{~N} / \mathrm{kg}$, which indicates that this animal was capable of full plantar weight-bearing stepping. Not every step was at full weight bearing, as the mean over 160 steps was $<4 \mathrm{~N} / \mathrm{kg}$ for the left hindlimb (mean $3.4 \mathrm{~N}$ ), although the mean indicated full weightbearing capability for the right hindlimb (mean $4.2 \mathrm{~N}$ ).

In graft cats, post-transection joint angles were compared with pre-transection to assess changes resulting from transection and graft therapy. Pre- and 5 week post-transection joint angles for the hip, knee, and ankle are reported in Figure 6. CONTROL cats were not included in these analyses, because the quality of stepping was so poor that post-transection angles had an unacceptably high variance for analysis. There was a significant reduction in hip range of motion in CHRONIC cats post-transection, falling from 54 degrees to 42 degrees as a result of decreased hip flexion. Knee angles were unchanged in all groups. Ankle angle, however, underwent significant changes after injury and recovery. All three graft groups had significant increases in total ankle range $(p>0.003)$. Additional analysis revealed significant increases in ankle maximum angle $(p<0.005)$ for the ACUTE, SUB-CHRONIC, and CHRONIC groups. There was a significant increase in ankle minimum angle in the ACUTE and CHRONIC groups $(p<0.033)$ after injury, although the increase was smaller than the increase in ankle maximum angle (12 degrees vs. 35 degrees).

\section{Maintenance of recovery over time in CHRONIC cats}

Cats in the CHRONIC group were observed for 12 weeks postgrafting, with kinematic recordings taken at 8 and 12 weeks post-grafting in addition to the recordings obtained at 5 weeks posttransection and 3 and 5 weeks post-grafting. An additional recording session occurred 1 week pre-grafting ( 5 weeks post-transection; at this time point, only two of the four CHRONIC cats were capable of stepping, and this was of low quality and minimal speeds [ $\leq 0.4 \mathrm{~m} / \mathrm{sec}]$ ). All but one CHRONIC cats had recovered some capacity for stepping 3 weeks post grafting $(\geq 0.6 \mathrm{~m} / \mathrm{sec})$, and by 5 weeks post-grafting, all CHRONIC cats were performing at least 10 plantar weight-bearing steps at all tested treadmill speeds from 0.3 to $0.8 \mathrm{~m} / \mathrm{sec}$.

A linear mixed model analysis was performed to determine the effects of time post-grafting on the kinematics of walking at a speed of $0.4 \mathrm{~m} / \mathrm{sec}$. Stance length remained at pre-transection levels through all post-grafting time points in the CHRONIC cats, with no significant effect of time post-grafting $(p=0.196)$. Figure 7 shows the stance length index at the $3,5,9$, and 12 week time points in CHRONIC cats. Stance length indices reach pre-transection levels 3 weeks after grafting, and were maintained until the final recording session, 12 weeks after grafting, with no indication of a decline although histological results presented subsequently indicate that cell neurotrophin production was undetectable by 5 weeks postgrafting. There was no significant change in swing height over time ( $p=0.096)$, nor was there a significant change in $\mathrm{D}_{\mathrm{a}}(p=0.441)$ or $\mathrm{D}_{\mathrm{p}}(p=0.989)$ over time.

\section{Histological evaluation}

Production of BDNF and NT-3 by autologous fibroblasts was measured by ELISA assays performed on the remaining cells not utilized for grafting. In cats SUB-CHRONIC-6, CHRONIC-2, and CHRONIC-4, all the cells were required for grafting and, therefore, no ELISA could be performed. For all other samples, production of BDNF and NT-3 from the remaining fibroblasts over a $24 \mathrm{~h}$ period was determined and reported as ng/mL/24 h/10 6 cells. Table 2 lists

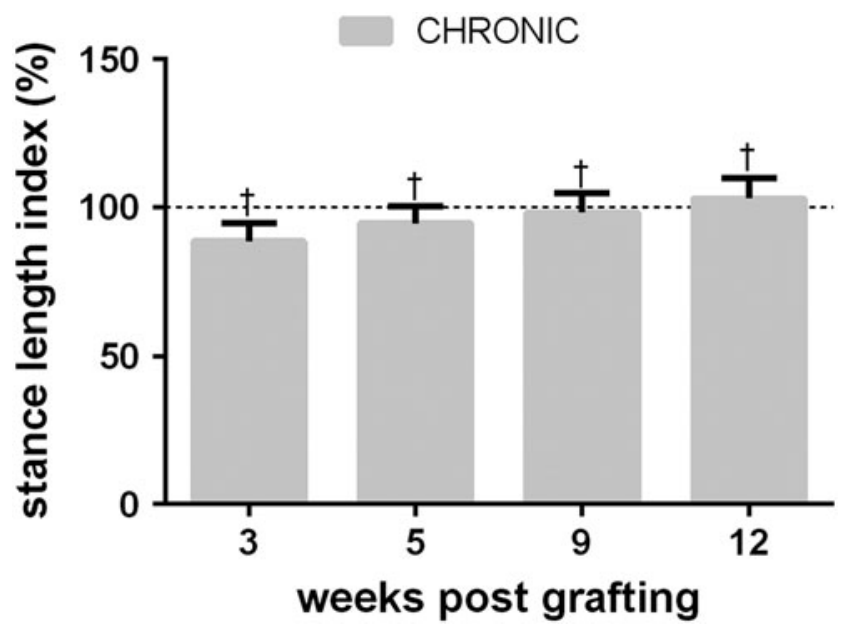

FIG. 7. Stance length index at 3-12 weeks post-grafting in CHRONIC graft cats walking at $0.4 \mathrm{~m} / \mathrm{sec}$. There was no significant change in stance length over the observed period of time. Stance length remained within $5 \%$ of the pre-transection means at all observed time points. †pre-transection group mean (100\%) falls within the group's $95 \%$ confidence interval (CI) post-injury

neurotrophin production levels for cells grafted into each cat Production values for neurotrophins are consistent with those in the literature for grafts with therapeutic properties ranging from 13 to $94 \mathrm{ng}$ of BDNF and from 0.5 to $48 \mathrm{ng}$ NT-3 $\left(\mathrm{mL} / 24 \mathrm{~h} / 10^{6}\right.$ cells). ${ }^{15,26-28}$ In some cases, production of one neurotrophin was high and the other was low; Student's $t$ tests were performed to determine if cats with grafts producing low-levels of neurotrophins showed diminished recovery. Analysis indicated that there was no effect of neurotrophin levels on average stance length $(t=-1.132$, $p=0.334)$ or maximum treadmill speed 3 weeks after grafting $(\mathrm{t}=$ $-1.755, p=0.169)$. Our results indicate that cats that received grafts producing low levels of one neurotrophin recovered stepping as

Table 2. BDNF and NT-3 Production in Grafted Cells

\begin{tabular}{|c|c|c|}
\hline Cat & $\begin{array}{c}\text { BDNF } \\
\left(n g / m L / 24 h / 10^{6}\right. \\
\text { cells })\end{array}$ & $\begin{array}{c}N T-3 \\
\left(\begin{array}{c}n g / m L / 24 h / 10^{6} \\
\text { cells })\end{array}\right.\end{array}$ \\
\hline ACUTE-1 & Not detectable & 2.70 \\
\hline ACUTE-2 & 5.23 & 3.37 \\
\hline SUB-CHRONIC-1 & 5.02 & 0.26 \\
\hline SUB-CHRONIC-2 & 0.07 & 8.75 \\
\hline SUB-CHRONIC-3 & 7.18 & 15.26 \\
\hline SUB-CHRONIC-4 & 23.13 & 240.59 \\
\hline SUB-CHRONIC-5 & 0.29 & 4.54 \\
\hline CHRONIC-1 & 5.38 & 81.13 \\
\hline CHRONIC-2 & Cells unavailable & Cells unavailable \\
\hline CHRONIC-3 & 1.57 & 9.5 \\
\hline CHRONIC-4 & Cells unavailable & Cells unavailable \\
\hline
\end{tabular}

Brain-derived neurotrophic factor (BDNF) and neurotrophic factor 3 (NT-3) production by the fibroblasts grafted into the spinal cord for each cat. Production varied greatly among batches, but there was no indication of a correlation with the locomotor recovery outcome among cats. In some animals, all the cells prepared were used and none were available to measure neurotrophins production from. Pre-implantation microscopic observation showed all cells expressing the green fluorescent protein (GFP) reporter. "Not detectable" indicates that the observed production was too low to quantify. "Cells unavailable" indicates that no cells remained for analysis after grafting. 


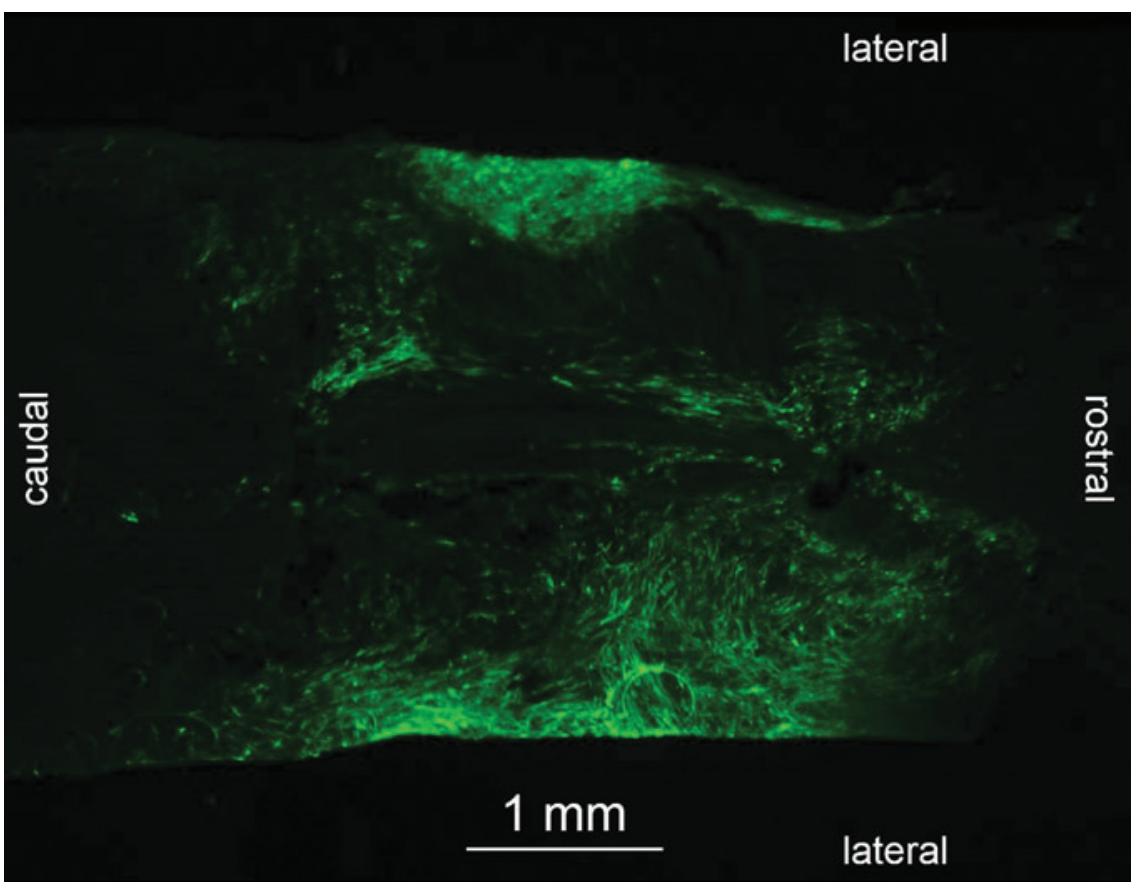

FIG. 8. Section of injury and cell graft site in cat SUB-CHRONIC-6 1 week after graft of modified fibroblasts in the horizontal plane near the central canal. The injury is a complete spinal cord transection at T11-T12, and the cells were grafted 2 weeks after the initial injury. Green fluorescent protein (GFP) fluorescing grafted fibroblasts are visible. Fluorescent cells were not visible in any spinal cord section of an animal euthanized $\geq 5$ weeks post-grafting. Color image is available online at www.liebertpub.com/neu

well as cats with high levels of both neurotrophins, consistent with previous work in our laboratory. ${ }^{21}$

After euthanisia, all spinal cords were removed for histological analysis. One cat (SUB-CHRONIC-6) was euthanized 1 week after cell graft to assess graft survival at an early time point; all other animals were euthanized 5 or 12 weeks post-grafting. Heavy GFP fluorescence was visible in the proximity of the lesion site in cat SUB-CHRONIC-6 (Fig. 8). No fluorescence was visible in the spinal cord of any cats euthanized 5 or 12 weeks after grafting. To ensure that GFP was not present at below-visible levels, we performed immunohistochemical staining for GFP. However, the secondary antibody could not be identified in any examined slices. Because none of our efforts could locate the graft, we are left to conclude that the grafted cells either dramatically downregulated neurotrophin production, or that the graft fibroblasts died or were targeted by immune cells for removal. Because fibroblasts naturally infiltrate a spinal lesion after injury, it is difficult, if not impossible, to conclusively identify the graft cells without the GFP marker. ${ }^{29,30}$

In some cases, fluid-filled cysts were visible in the lesion area, which is consistent with our previous transplant work in cats. ${ }^{12}$ Figure 9 shows Nissl-myelin- stained example lesion sites from a cat in each group, with cysts visible, in some cases. No axon regeneration through the lesion was visible in these or any other sections. Figure 10 shows fluorescent stained sections next to adjacent Nisslmyelin sections. Both rostral and caudal axons traveled into the graft, but there was no evidence of axons crossing the lesion in any animal. None of the histological analyses indicated quantifiable regeneration of neurons through the lesion site at any of the time points investigated (1,5, and 12 weeks post-grafting), similar to our earlier results with neurotrophin-producing xenografts. ${ }^{12}$

Overall, these results indicate that neurotrophin delivery from grafted cells is limited to the first 5 weeks when using autologous cells in cats, and that locomotor recovery does not occur through a mechanism involving axonal growth through the injury site.

\section{Discussion}

\section{Summary of the study's findings}

Our study has three primary findings: 1) autologous fibroblasts, modified to produce the neurotrophins BDNF and NT-3, promote recovery of stepping in the spinal cat; 2) 2- or 6-week delayed delivery of neurotrophin-producing grafts promotes recovery of stepping as well as acutely delivered grafts; and 3) the recovery from graft therapy is maintained even after downregulation of neurotrophin production. These results clear several hurdles to turning neurotrophin-producing grafts into a clinically viable therapy.

This is the first study to report motor recovery after treatment with autologous, neurotrophin-producing fibroblasts in a feline complete spinal transection model. The only previous results with autologous grafting of neurotrophin-producing fibroblasts in a primate hemisection model of SCI examined axonal growth; however, no functional benefits were reported. ${ }^{31}$ As in the primate, we observed no axonal regeneration through the injury site filled with autologous neurotrophin-producing grafts, confirming earlier results with neurotrophin-producing xenografts in cats, ${ }^{12,21}$ or with delivery of BDNF into the transection site in rats. ${ }^{27}$

Recovery of stance length and swing height was similar to or better than what can be obtained with xenografts or BWST. 1,12,32 We did observe changes in the ankle range, maximum, and minimum for all graft groups. The change appears to be the result of stronger ankle extension and weaker ankle flexion, and has been previously reported. ${ }^{33}$ Whereas Petruska and coworkers have reported that enhanced monosynaptic gastrocnemius excitatory postsynaptic potentials (EPSPs) are related to improved recovery 

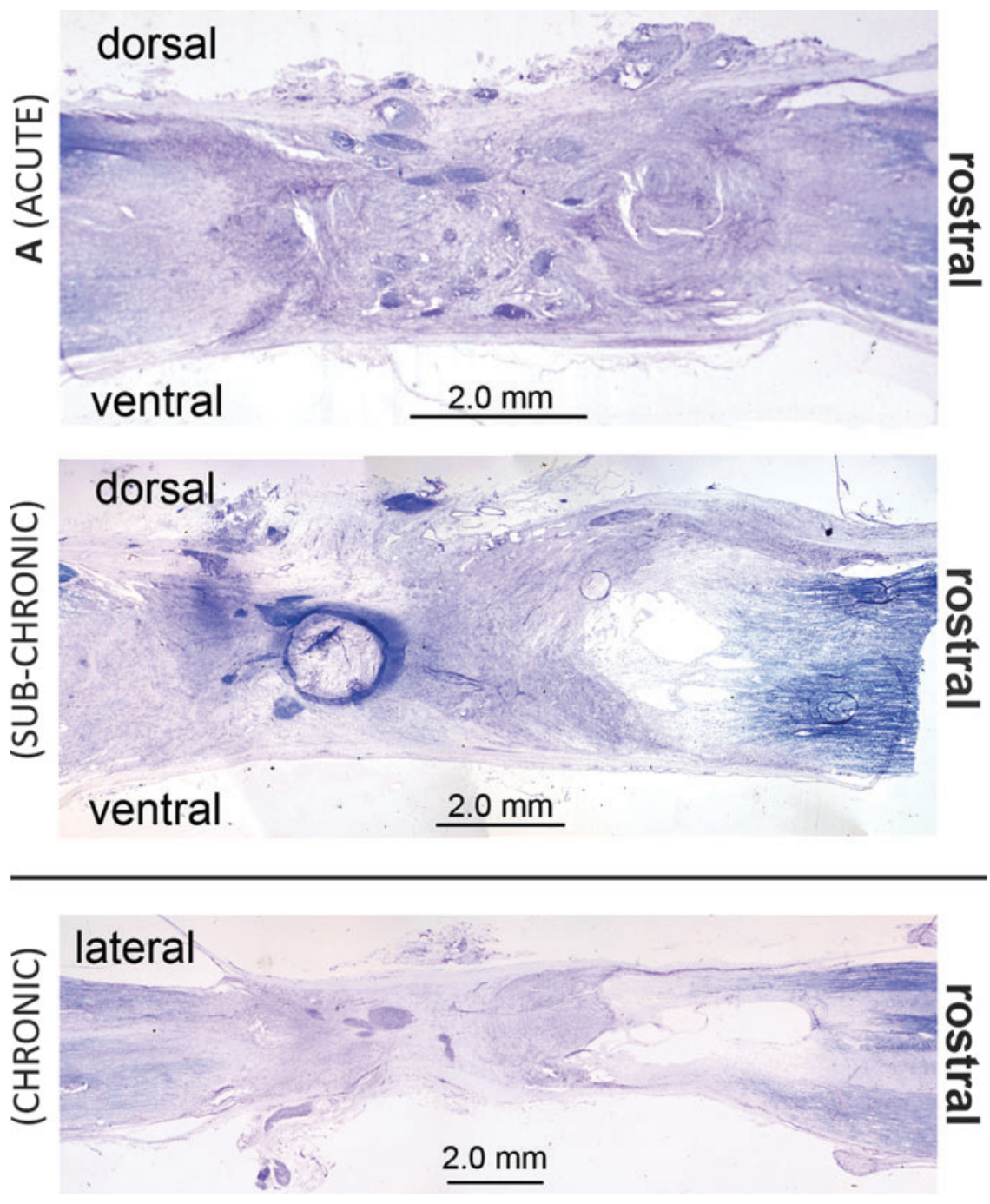

FIG. 9. Nissl-myelin stained sections of the lesion site in graft cats. (A) Sagittal section from the midline of the lesion site in cat ACUTE2. The darkened purple clusters in the center of the lesion are groups of small, undifferentiated cells. (B) Sagittal section at the midline of the lesion site in cat SUB-CHRONIC-3. (C) Horizontal section near the center of the lesion in cat CHRONIC-1. No axonal sparing or growth across the lesion could be identified in Nissl-myelin stained sections. Cysts can be seen in images $\mathbf{B}$ and $\mathbf{C}$ on the rostral end of the lesion; the presence of cysts has been noted in previous work, ${ }^{12}$ and was not linked to differences in kinematic recovery. Color image is available online at www.liebertpub.com/neu

with training in rats, ${ }^{34}$ Côte and coworkers reported that training reduced monosynaptic ankle extensor EPSPs in cats. ${ }^{35}$ Therefore, whereas an enhanced ankle extensor stretch reflex may have contributed to the hyperextension observed, previous reports show that recovery (through training) may involve increase or decrease activity in the ankle extensor stretch reflex. It is unlikely that hyperextension is related to greater muscle atrophy in the ankle flexors, as muscle weight has been shown to scale within a limb following spinal injury. ${ }^{36}$

Our results with 6 weeks delayed grafting were also surprising, considering the previous literature on the limited efficacy of neurotrophin-producing transplants in chronic SCI. Studies in rats ${ }^{15,37,38}$ have reported that extended delays before the beginning of treatment negatively impact the efficacy of treatment and lead to diminished recovery of motor capability compared with acutely treated animals. It should be noted that many of those studies use an incomplete model of SCI, ${ }^{15,37-40}$ and measures of recovery typically included balance tests, forelimb-hindlimb coordination, and other measures that are highly dependent on supraspinal input. In our full transection model, and using weight-bearing stepping as our measure of recovery, the chronic-delay group readily recovered treadmill stepping as well as acutely grafted animals.

Finally, our histological examination showed no detectable production of neurotrophins within the cavity site 5 or 12 weeks postgrafting, although the fibroblasts' reporter gene was readily apparent 1 week post-grafting, indicating that the cells survived grafting and produced neurotrophins for at least 1 week following delivery. Despite the apparent lack of neurotrophin production by week 5 , our graft animals demonstrated plantar weight-bearing stepping at up to $0.8 \mathrm{~m} / \mathrm{sec}$, even at 12 weeks post-grafting. Neurotrophin production by genetically modified fibroblasts is significantly downregulated in vivo by 8 weeks, even in immunosuppressed rats that received an allograft. ${ }^{16,28}$ The downregulation observed in our cats was much more pronounced; however, immunostaining showed no GFP at 5 or 12 weeks post-grafting in any of our animals. This pronounced downregulation may be the result of a more aggressive immune system 


\section{rostral}
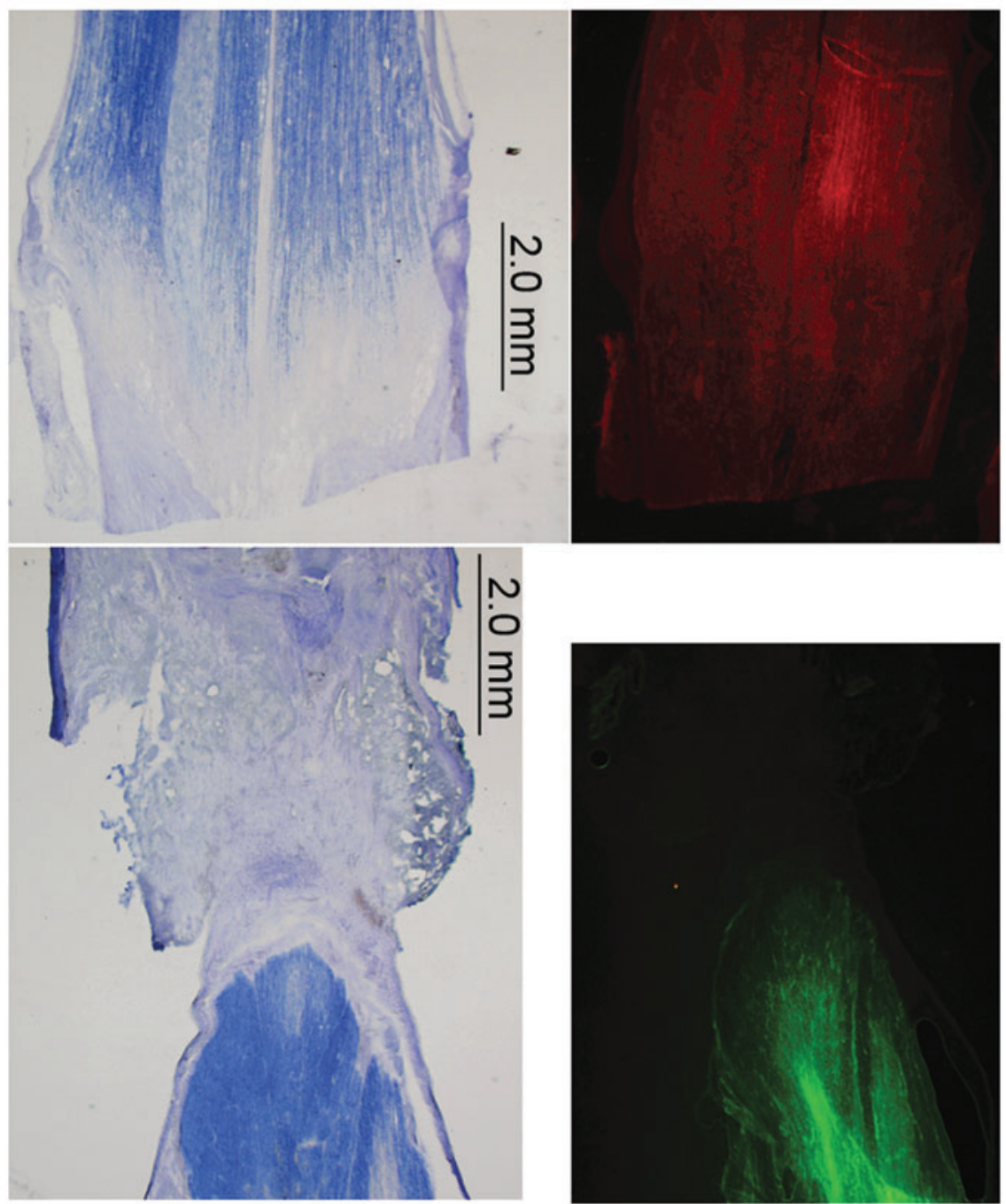

\section{caudal}

FIG. 10. Axon tracing in a chronic delayed graft cat (CHRONIC-3) from a slice at approximately the dorsal-ventral midline of the spinal cord. The rostral end of the lesion site, $\sim 1 \mathrm{~mm}$ from the edge of the lesioned tissue, was injected with micro-ruby tracer (3000MW, D7162) and the caudal end was injected with micro-emerald (3000MW, D7156). All images are from horizontal sections near the center of the spinal cord. Fluorescent images are from sections adjacent to the corresponding Nissl-myelin- stained sections. Axons travel to the lesion edge but stop before entering or crossing the lesion. There was no evidence of emerald-stained axons in the rostral segment or of ruby-stained axons in the caudal segment. Sections immediately adjacent (dorsal and ventral) were also negative of any indication of growth through the lesion. In addition, no evidence of crossing was visible in sections rostral and caudal to the ones presented. Color image is available online at www.liebertpub.com/neu

response to cells producing a human protein in an outbred species compared with the immune response in the more inbred rodents.

\section{Potential mechanisms for locomotor recovery}

Our study does not provide a clear mechanism for the recovery of stepping resulting from our graft, although we can speculate on the mechanism based on the relationship between exercise and neurotrophins, and our previous neuromechanical system modeling results. Spinal neurotrophin levels are modified by exercise, with BDNF showing an increase, ${ }^{41}$ whereas NT-3 undergoes an early decrease but increases after several days of training. ${ }^{42}$ Neurotrophins have enhancing effects on synaptic efficacy, ${ }^{43,44}$ and modeling results show recovery of locomotion in a spinalized neuromechanical model of the cat hindlimb when the strength of the synaptic connections between the model's spinal cord interneurons ${ }^{45}$ and the strength of the muscles' proprioceptive feedback are increased. ${ }^{46}$ It is most likely that the differential and dose-dependent effects of BDNF and NT-3 modulate motoneuron activity in our model, with exogenous BDNF and NT-3 mirroring the increase in BDNF and NT-3 that results from training and exercise. ${ }^{40,47-49}$ Because our grafts contribute BDNF and NT-3 to the spinal circuitry, they may be activating these same mechanisms.

Although our grafts were inserted at a distance from the locomotor centers, neurotrophic factors are readily transported transsynaptically, ${ }^{50-52}$ and ascending sensory tracts may uptake and 
retrogradely transport neurotrophins to the lumbar spinal cord, increasing the synaptic efficiency of sensory input to the stepping circuitry. Alternatively, neurotrophins transported trans-synaptically to interneurons of the spinal gray matter may increase the activity state of the stepping circuitry. We believe that the neurotrophins produced by our graft are transported throughout the spinal cord and are responsible for the improvement of locomotion

Comparisons between the time course of neurotrophin delivery from our graft, and the time course of motor recovery and retention with training lead to an interesting paradox that warrants further exploration in longer-term experiments. Although treadmill training is effective at promoting recovery of stepping, training must be continuous if the recovery is to be maintained. de Leon and coworkers showed that in the absence of training, locomotor capability declines significantly 12 weeks after the cessation of training, with a nonsignificant trend beginning 6 weeks after injury. ${ }^{32}$ Our cats recovered stepping to normal levels and maintained this for 12 weeks after injury, no less than 7 weeks after neurotrophin production could no longer be detected. We cannot absolutely state that our cats will maintain similar levels of recovery at further time points; however, the absence of a trend of decreasing stance length combined with similar results from previous work in the laboratory utilizing xenografts ${ }^{12}$ suggests that delivery of neurotrophins need not be chronic to retain recovery. Temporary release of the neurotrophins may promote stepping while avoiding the "spasticity syndrome" reported in rats receiving prolonged delivery of BDNF and NT-3 via adenoassociated virus (AAV) vector injections. ${ }^{47}$

We believe that this study shows promising results for the future of neurotrophin delivery as a viable therapy for patients with both acute and chronic spinal injuries. To date, no other work has as thoroughly explored the value of autologous, neurotrophin- producing grafts, or the value of this manner of graft therapy in a chronic SCI model. This study observed 14 animals in four experimental groups for 5-12 weeks, a large group in comparison with most cat studies. ${ }^{12,33,53-56}$ The work presented here raises the translational potential of neurotrophin treatments for humans, although a number of additional avenues need to be explored. Future studies should extend the analysis period to gain a more complete insight into the long-term effects of therapy and expand it into incomplete SCI models where the potential but debated role of BDNF in nociception may lead to unwanted side effects. ${ }^{57,58}$

\section{Latent capability of the locomotor circuitry}

Our results confirm the robustness and autonomous capability of the lumbar locomotor circuitry in the absence of any supraspinal input in the spinal cat model. In the complete spinal cat, the locomotor circuitry can be re-engaged with locomotor training, $1,2,32,33,55,59-61$ epidural stimulation, ${ }^{56}$ or neurotrophin delivery. ${ }^{12,21}$ Although delivered to the injury site, our results do not appear to be based on axonal growth through the transplant, but rather on neurotrophin effects on the lumbar circuitry. These results suggest that whereas chronic delays have a negative effect on the regenerative capacity of the spinal cord, delays have no apparent effect on the latent capabilities of the locomotor circuitry. Further, our results suggest that at a chronic time point, when the post-injury regenerative and degenerative processes are coming to an end, ${ }^{62,63}$ the locomotor circuitry can still be activated and is still readily capable of generating plantarweight-bearing stepping.

In summary, our results show that autologous neurotrophinproducing grafts are effective at promoting recovery of locomotion even in conditions of chronic injury. These results establish realistic and promising protocols for clinical application to people with SCI.

\section{Acknowledgments}

We thank Dr. Timothy B. Himes and Maryla Obracka for technical assistance and support. In addition, we thank Kassi Miller and Jennifer Dashkova for their assistance with animal training. This research was supported by National Institute of Neurological Disorders and Stroke Grant NIH NS074406 and the National Institute of Biomedical Imaging and Bioengineering Grant NIH EB012855.

\section{Author Disclosure Statement}

No competing financial interests exist.

\section{References}

1. Belanger, M., Drew, T., Provencher, J., and Rossignol, S. (1996). A comparison of treadmill locomotion in adult cats before and after spinal transection. J. Neurophysiol. 76, 471-491.

2. Lovely, R.G., Gregor, R.J., Roy, R.R., and Edgerton, V.R. (1986). Effects of training on the recovery of full-weight-bearing stepping in the adult spinal cat. Exp. Neurol. 92, 421-435.

3. Behrman, A.L., Nair, P.M., Bowden, M.G., Dauser, R.C., Herget, B.R., Martin, J.B., Phadke, C.P., Reier, P.J., Senesac, C.R., Thompson, F.J., and Howland, D.R. (2008). Locomotor training restores walking in a nonambulatory child with chronic, severe, incomplete cervical spinal cord injury. Phys. Ther. 88, 580-590.

4. Harkema, S.J. (2008). Plasticity of interneuronal networks of the functionally isolated human spinal cord. Brain Res. Rev. 57, 255-264.

5. Wirz, M., Colombo, G. and Dietz, V. (2001). Long term effects of locomotor training in spinal humans. J. Neurol. Neurosurg. Psychiatry 71, 93-96.

6. Dobkin, B.H., Harkema, S., Requejo, P., and Edgerton, V.R. (1995). Modulation of locomotor-like EMG activity in subjects with complete and incomplete spinal cord injury. J. Neurol. Rehabil. 9, 183-190.

7. Dietz, V. (2009). Body weight supported gait training: from laboratory to clinical setting. Brain Res. Bull. 78, I-VI.

8. Harkema, S., Gerasimenko, Y., Hodes, J., Burdick, J., Angeli, C., Chen, Y., Ferreira, C., Willhite, A., Rejc, E., Grossman, R.G., and Edgerton, V.R. (2011). Effect of epidural stimulation of the lumbosacral spinal cord on voluntary movement, standing, and assisted stepping after motor complete paraplegia: a case study. Lancet 377, 1938-1947.

9. Edgerton, V.R., and Harkema, S. (2011). Epidural stimulation of the spinal cord in spinal cord injury: current status and future challenges. Expert Rev. Neurother. 11, 1351-1353.

10. Angeli, C.A., Edgerton, V.R., Gerasimenko, Y.P., and Harkema, S.J. (2014). Altering spinal cord excitability enables voluntary movements after chronic complete paralysis in humans. Brain 137, 1394-1409.

11. Minassian, K., Hofstoetter, U.S., Danner, S.M., Mayr, W., Bruce, J.A., McKay, W.B., and Tansey, K.E. (2016). Spinal rhythm generation by step-induced feedback and transcutaneous posterior root stimulation in complete spinal cord-injured individuals. Neurorehabil. Neural Repair 30, 233-243

12. Boyce, V.S., Tumolo, M., Fischer, I., Murray, M., and Lemay, M.A. (2007). Neurotrophic factors promote and enhance locomotor recovery in untrained spinalized cats. J. Neurophysiol. 98, 19881996.

13. de Leon, R.D. (2007). Could neurotrophins replace treadmill training as locomotor therapy following spinal cord injury? Focus on "neurotrophic factors promote and enhance locomotor recovery in untrained spinalized cats". J. Neurophysiol. 98, 1845-1846.

14. Mitsui, T., Fischer, I., Shumsky, J.S., and Murray, M. (2005). Transplants of fibroblasts expressing BDNF and NT-3 promote recovery of bladder and hindlimb function following spinal contusion injury in rats. Exp. Neurol. 194, 410-431.

15. Tobias, C.A., Shumsky, J.S., Shibata, M., Tuszynski, M.H., Fischer, I., Tessler, A., and Murray, M. (2003). Delayed grafting of BDNF and 
NT-3 producing fibroblasts into the injured spinal cord stimulates sprouting, partially rescues axotomized red nucleus neurons from loss and atrophy, and provides limited regeneration. Exp. Neurol. 184, 97-113.

16. Liu, Y., Himes, B.T., Tryon, B., Moul, J., Chow, S.Y., Jin, H., Murray, M., Tessler, A., and Fischer, I. (1998). Intraspinal grafting of fibroblasts genetically modified by recombinant adenoviruses. NeuroReport 9, 1075-1079.

17. Himes, B.T., Liu, Y., Solowska, J.M., Snyder, E.Y., Fischer, I., and Tessler, A. (2001). Transplants of cells genetically modified to express neurotrophin-3 rescue axotomized Clarke's nucleus neurons after spinal cord hemisection in adult rats. J. Neurosci. Res. 65, 549-564.

18. Adami, J., Gabel, H., Lindelof, B., Ekstrom, K., Rydh, B., Glimelius, B., Ekbom, A., Adami, H.O., and Granath, F. (2003). Cancer risk following organ transplantation: a nationwide cohort study in Sweden. Br. J. Cancer 89, 1221-1227.

19. Jensen, P., Hansen, S., Møller, B., Leivestad, T., Pfeffer, P., Geiran, O., Fauchald, P., and Simonsen, S. (1999). Skin cancer in kidney and heart transplant recipients and different long-term immunosuppressive therapy regimens. J. Am. Acad. Dermatol. 40, 177-186.

20. Blesch, A. (2004). Lentiviral and MLV based retroviral vectors for ex vivo and in vivo gene transfer. Methods 33, 164-172.

21. Ollivier-Lanvin, K., Fischer, I., Tom, V., Houle, J.D., and Lemay, M.A. (2015). Either brain-derived neurotrophic factor or neurotrophin3 only neurotrophin-producing grafts promote locomotor recovery in untrained spinalized cats. Neurorehabil. Neural Repair 29, 90-100.

22. Goslow, G.E., Reinking, R.M., and Stuart, D.G. (1973). The cat step cycle: Hind limb joint angles and muscle lengths during unrestrained locomotion. J. Morphol. 141, 1-41.

23. Macpherson, J.M. (1988). Strategies that simplify the control of quadrupedal stance. I. Forces at the ground. J. Neurophysiol. 60, 204217.

24. Pratt, C.A., Fung, J., and Macpherson, J.M. (1994). Stance control in the chronic spinal cat. J. Neurophysiol. 71, 1981-1985.

25. Manter, J.T. (1938). The dynamics of quadrupedal walking. J. Exp. Biol. 15, 522-540.

26. Boyce, V.S. (2006). Treadmill Locomotor Training and Neurotrophic Factors: Their Effect on Locomotor Recovery and Spinal Modularity in the Chronic Spinal Cat. Neurobiology \& Anatomy, Drexel University College of Medicine: Philadelphia.

27. Jakeman, L.B., Wei, P., Guan, Z., and Stokes, B.T. (1998). Brainderived neurotrophic factor stimulates hindlimb stepping and sprouting of cholinergic fibers after spinal cord injury. Exp. Neurol. 154, 170-184.

28. Liu, Y., Kim, D., Himes, B.T., Chow, S.Y., Schallert, T., Murray, M., Tessler, A., and Fischer, I. (1999). Transplants of fibroblasts genetically modified to express BDNF promote regeneration of adult rat rubrospinal axons and recovery of forelimb function. J. Neurosci. 19, 4370-4387.

29. Soderblom, C., Luo, X., Blumenthal, E., Bray, E., Lyapichev, K., Ramos, J., Krishnan, V., Lai-Hsu, C., Park, K.K., Tsoulfas, P., and Lee, J.K. (2013). Perivascular fibroblasts form the fibrotic scar after contusive spinal cord injury. J. Neurosci. 33, 13,882-13,887.

30. Göritz, C., Dias, D.O., Tomilin, N., Barbacid, M., Shupliakov, O., and Frisén, J. (2011). A pericyte origin of spinal cord scar tissue. Science 333, 238-242.

31. Brock, J.H., Rosenzweig, E.S., Blesch, A., Moseanko, R., Havton, L.A., Edgerton, V.R., and Tuszynski, M.H. (2010). Local and remote growth factor effects after primate spinal cord injury. J. Neurosci. 30, 9728-9737.

32. de Leon, R.D., Hodgson, J.A., Roy, R.R., and Edgerton, V.R. (1999). Retention of hindlimb stepping ability in adult spinal cats after the cessation of step training. J. Neurophysiol. 81, 85-94.

33. Barbeau, H., and Rossignol, S. (1987). Recovery of locomotion after chronic spinalization in the adult cat. Brain Res. Rev. 412, 84-95.

34. Petruska, J.C., Ichiyama, R.M., Jindrich, D.L., Crown, E.D., Tansey, K.E., Roy, R.R., Edgerton, V.R., and Mendell, L.M. (2007). Changes in motoneuron properties and synaptic inputs related to step training after spinal cord transection in rats. J. Neurosci. 27, 4460-4471.

35. Côte, M.-P., Menard, A., and Gossard, J.-P. (2003). Spinal cats on the treadmill: changes in load pathways. J. Neurosci. 23, 27892796.
36. Giszter, S.F., Hockensmith, G., Ramakrishnan, A., and Udoekwere, U.I. (2010). How spinalized rats can walk: biomechanics, cortex, and hindlimb muscle scaling-implications for rehabilitation. Ann. N. Y. Acad. Sci. 1198, 279-293.

37. Norrie, B.A., Nevett-Duchcherer, J.M., and Gorassini, M.A. (2005). Reduced functional recovery by delaying motor training after spinal cord injury. J. Neurophysiol. 94, 255-264.

38. Shumsky, J.S., Tobias, C.A., Tumolo, M., Long, W.D., Giszter, S.F., and Murray, M. (2003). Delayed transplantation of fibroblasts genetically modified to secrete BDNF and NT-3 into a spinal cord injury site is associated with limited recovery of function. Exp. Neurol. 184, 114-130.

39. Karimi-Abdolrezaee, S., Eftekharpour, E., Wang, J., Morshead, C.M., and Fehlings, M.G. (2006). Delayed transplantation of adult neural precursor cells promotes remyelination and functional neurological recovery after spinal cord injury. J. Neurosci. 26, 33773389 .

40. Plant, G.W., Christensen, C.L., Oudega, M., and Bunge, M.B. (2003). Delayed transplantation of olfactory ensheathing glia promotes sparing/regeneration of supraspinal axons in the contused adult rat spinal cord. J. Neurotrauma 20, 1-16.

41. Ying, Z., Roy, R.R., Edgerton, V.R., and Gómez-Pinilla, F. (2005). Exercise restores levels of neurotrophins and synaptic plasticity following spinal cord injury. Exp. Neurol. 193, 411-419.

42. Gómez-Pinilla, F., Ying, Z., Opazo, P., Roy, R.R., and Edgerton, V.R. (2001). Differential regulation by exercise of BDNF and NT-3 in rat spinal cord and skeletal muscle. Eur. J. Neurosci. 13, 10781084

43. Poo, M.M. (2001). Neurotrophins as synaptic modulators. Nat. Rev. Neurosci. 2, 24-32.

44. Mendell, L.M., Munson, J.B., and Arvanian, V.L. (2001). Neurotrophins and synaptic plasticity in the mammalian spinal cord. J. Physiol. 533, 91-97.

45. Lemay, M.A., Markin, S.N., Klishko, A.N., Shevtsova, N.A., OllivierLanvin, K., Prilutsky, B.I., and Rybak, I. (2009). Afferent neuroplasticity as a possible mechanism for the locomotor recovery seen with neurotrophin transplants: insights from modeling studies, in: Biomedical Engineering Society Annual Meeting. IEEE: Pittsburgh, PA.

46. Markin, S.N., Klishko, A.N., Shevtsova, N.A., Lemay, M.A., Prilutsky, B.I., and Rybak, I.A. (2010). Afferent control of locomotor CPG: insights from a simple neuromechanical model. Ann. N. Y. Acad. Sci. 1198, 21-34.

47. Boyce, V.S., Park, J., Gage, F.H., and Mendell, L.M. (2012). Differential effects of brain-derived neurotrophic factor and neurotrophin-3 on hindlimb function in paraplegic rats. Eur. J. Neurosci. $35,221-232$.

48. Friedman, B., Kleinfeld, D., Ip, N., Verge, V., Moulton, R., Boland, P., Zlotchenko, E., Lindsay, R., and Liu, L. (1995). BDNF and NT-4/5 exert neurotrophic influences on injured adult spinal motor neurons. J. Neurosci. 15, 1044-1056.

49. Petruska, J.C., Kitay, B., Boyce, V.S., Kaspar, B.K., Pearse, D.D., Gage, F.H., and Mendell, L.M. (2010). Intramuscular AAV delivery of NT-3 alters synaptic transmission to motoneurons in adult rats. Eur. J. Neurosci. 32, 997-1005.

50. Altar, C.A., and DiStefano, P.S. (1998). Neurotrophin trafficking by anterograde transport. Trends Neurosci. 21, 433-437.

51. DiStefano, P.S., Friedman, B., Radziejewski, C., Alexander, C., Boland, P., Schick, C.M., Lindsay, R.M., and Wiegand, S.J. (1992). The neurotrophins BDNF, NT-3, and NGF display distinct patterns of retrograde axonal transport in peripheral and central neurons. Neuron 8, 983-993.

52. von Bartheld, C.S. (2004). Axonal transport and neuronal transcytosis of trophic factors, tracers, and pathogens. J. Neurobiol. 58, 295-314.

53. Howland, D.R., Bregman, B.S., Tessler, A., and Goldberger, M.E. (1995). Development of locomotor behavior in the spinal kitten. Exp. Neurol. 135, 108-122.

54. Barbeau, H., and Rossignol, S. (1990). The effects of serotonergic drugs on the locomotor pattern and on cutaneous reflexes of the adult chronic spinal cat. Brain Res. 514, 55-67.

55. de Leon, R.D., Hodgson, J.A., Roy, R.R., and Edgerton, V.R. (1998). Locomotor capacity attributable to step training versus spontaneous recovery after spinalization in adult cats. J. Neurophysiol. 79, 13291340. 
56. Gerasimenko, Y.P., Avelev, V.D., Nikitin, O.A., and Lavrov, I.A. (2003). Initiation of locomotor activity in spinal cats by epidural stimulation of the spinal cord. Neurosci. Behav. Physiol. 33, 247-254.

57. Constandil, L., Aguilera, R., Goich, M., Hernández, A., Alvarez, P. Infante, C., and Pelissier, T. (2011). Involvement of spinal cord BDNF in the generation and maintenance of chronic neuropathic pain in rats. Brain Res. Bull. 86, 454-459.

58. Weishaupt, N., Blesch, A., and Fouad, K. (2012). BDNF: The career of a multifaceted neurotrophin in spinal cord injury. Exp. Neurol. 238, 254-264.

59. Lovely, R.G., Gregor, R.J., Roy, R.R., and Edgerton, V.R. (1990). Weight-bearing hindlimb stepping in treadmill-exercised adult spinal cats. Brain Res. 514, 206-218.

60. Rossignol, S., and Bouyer, L. (2004). Adaptive mechanisms of spinal locomotion in cats. Integr. Comp. Biol. 44, 71-79.

61. Belanger, M., Drew, T., and Rossignol, S. (1988). Spinal locomotion: a comparison of the kinematics and the electromyographic activity in the same animal before and after spinalization. Acta Biol. Hung. 39, 151-154.

62. Ramon, Y., and Cajal, S. (1928). Degeneration \& Regeneration of the Nervous System, Vol 1. Hafner Publishing Co.: New York.
63. Ramon, Y., and Cajal, S. (1928). Degeneration \& Regeneration of the Nervous System, Vol 2. Hafner Publishing Co.: New York.

64. Corbee, R.J., Maas, H., Doornenbal, A., and Hazewinkel, H.A. (2014). Forelimb and hindlimb ground reaction forces of walking cats: assessment and comparison with walking dogs. Vet. J. 202, 116-127.

65. Gregor, R.J., Smith, D.W., and Prilutsky, B.I. (2006). Mechanics of slope walking in the cat: quantification of muscle load, length change, and ankle extensor EMG patterns. J. Neurophysiol. 95, 1397-1409.

Address correspondence to:

Michel A. Lemay, PhD

Department of Bioengineering

College of Engineering

Temple University

Engineering Building Room 808

Philadelphia, PA 19122

E-mail: mlemay@temple.edu 